\title{
Black Holes as Lumps of Fluid
}

\author{
Marco M. Caldarelli, ${ }^{1,2}$ Óscar J. C. Dias, ${ }^{1,3}$ Roberto Emparan, ${ }^{1,4}$ Dietmar Klemm, ${ }^{5}$ \\ ${ }^{1}$ Departament de Física Fonamental, Universitat de Barcelona, \\ Marti i Franquès 1, E-08028 Barcelona \\ ${ }^{2}$ Instituut voor Theoretische Fysica, Katholieke Universiteit Leuven, \\ Celestijnenlaan 200D B-3001 Leuven, Belgium \\ ${ }^{3}$ Dept. de Física e Centro de Física do Porto, Faculdade de Ciências da Universidade do Porto, \\ Rua do Campo Alegre 687, 4169 - 007 Porto, Portugal \\ ${ }^{4}$ Institució Catalana de Recerca i Estudis Avançats (ICREA), \\ Passeig Lluís Companys 23, E-08010 Barcelona, Spain \\ ${ }^{5}$ Dipartimento di Fisica dell'Università di Milano, \\ Via Celoria 16, I-20133 Milano and \\ INFN, Sezione di Milano, Via Celoria 16, I-20133 Milano. \\ caldarelli@ub.edu, oscar.dias@fc.up.pt, emparan@ub.edu, dietmar.klemm@mi.infn.it
}

\begin{abstract}
The old suggestive observation that black holes often resemble lumps of fluid has recently been taken beyond the level of an analogy to a precise duality. We investigate aspects of this duality, and in particular clarify the relation between area minimization of the fluid vs. area maximization of the black hole horizon, and the connection between surface tension and curvature of the fluid, and surface gravity of the black hole. We also argue that the Rayleigh-Plateau instability in a fluid tube is the holographic dual of the Gregory-Laflamme instability of a black string. Associated with this fluid instability there is a rich variety of phases of fluid solutions that we study in detail, including in particular the effects of rotation. We compare them against the known results for asymptotically flat black holes finding remarkable agreement. Furthermore, we use our fluid results to discuss the unknown features of the gravitational system. Finally, we make some observations that suggest that asymptotically flat black holes may admit a fluid description in the limit of large number of dimensions.
\end{abstract}




\section{Contents}

1 Introduction $\quad 2$

2 Hydrodynamic description of deconfined plasma lumps

2.1 Navier-Stokes and Young-Laplace equations . . . . . . . . . . . . . . . 4

2.2 Stationary plasma configurations . . . . . . . . . . . . . . 5

3 Variational principles for equilibrium plasma configurations 8

3.1 Relativistic soap bubbles and second law of thermodynamics . . . . . . . . . 8

3.2 Equivalent variational principles for spinning lumps . . . . . . . . . . . . . . 10

4 Static and rotating equilibrium plasma configurations $\quad 11$

4.1 General formulation of the stationary and axisymmetric case . . . . . . . . . . 11

4.2 Static plasma lumps . . . . . . . . . . . . . . . . . . 12

4.3 Rotating plasma lumps . . . . . . . . . . . . . . . . 14

5 Rayleigh-Plateau instability of plasma tubes $\quad 18$

5.1 Unstable threshold mode from entropy or potential energy variation . . . . . . . . . 19

5.1.1 Threshold mode for static and boosted plasmas . . . . . . . . . . . . . . . 19

5.1.2 Threshold mode for rotating plasma and non-axisymmetric modes . . . . . . 19

5.2 Rayleigh-Plateau dispersion relation . . . . . . . . . . . . . . . . 20

5.2.1 Perturbations of static tubes in any dimension . . . . . . . . . . . . 22

5.2 .2 Rotating and boosted plasma lumps . . . . . . . . . . . . . . 24

5.2 .3 Viscosity . . . . . . . . . . . . . . . . . . . 26

\begin{tabular}{ll|l}
6 & Regime of validity & $\mathbf{2 7}$ \\
\hline
\end{tabular}

7 Rayleigh-Plateau and Gregory-Laflamme 2

8 The black hole/fluid analogy revisited 


\section{Introduction}

The tantalizing similarities between, on the one hand, the physics of black holes, and on the other hand, the properties of soap bubbles and fluid lumps, have been observed from old and indeed motivated approaches like the membrane paradigm. A black hole is bounded by a smooth horizon with uniform surface gravity, in analogy with the surface of a fluid droplet which, under the effect of surface tension, assumes a smooth shape with constant mean curvature. The negative specific heat of a black hole, and its concomitant Hawking radiation, bear a resemblance to the evaporation of a fluid droplet. More recently, refs. [1, 2, 3] pointed out a striking similarity between the RayleighPlateau instability of a column of fluid and the Gregory-Laflamme instability of black strings 4, 5].

However, when one tries to take these observations beyond the level of mere analogies, a number of apparent difficulties arise. A soap bubble, or a lump of fluid - at least a static one - strives to minimize its surface area, whereas a black hole tends to maximize the area of its horizon. It is also difficult to make precise in which sense the surface gravity relates to the surface tension of the fluid, or whether it should be related instead to the mean curvature of the fluid surface. Underlying these difficulties is the question of whether there is any regime in which the gravitational dynamics of black hole horizons can be precisely mapped into the dynamics of fluids.

The issue can be decisively settled within the context of the AdS/CFT correspondence, which turns the analogy into a precise duality at least for a certain class of black holes in Anti-de Sitter space. Refs. [6, 7] have shown that large black holes in AdS gravity can be mapped to solutions of the Navier-Stokes equations of a conformal fluid. In this framework, large black holes in a spacetime asymptotic to AdS with boundary $\mathcal{B}$ correspond to fluid configurations filling the spacetime $\mathcal{B}$. However, the physics of these black holes differs significantly from the physics of, say, the Schwarzschild black hole in asymptotically flat space. For instance, large AdS black holes have positive specific heat, and thus do not disappear via Hawking emission. In fact, the analogies mentioned above seem to lose some of their motivation since the fluid duals to these black holes do not have a bounding surface. Also, black strings exist in $\operatorname{AdS}_{d}$, with horizon topology $\mathbb{R} \times S^{d-2}$ extended in a direction along an asymptotic boundary of topology $\mathbb{R}_{t} \times \mathbb{R} \times S^{d-2}$ [8, 9, 10], but when these black strings are large they do not suffer from the Gregory-Laflamme instability [11]. In dual terms, they correspond to a fluid filling the boundary. This fluid is locally stable under plasma oscillations (i.e., sound waves propagating in the fluid) so in the long-wavelength approximation these configurations are stable. Thus, these set-ups do not seem adequate to make precise the connection between black holes and fluid lumps, and in particular the instabilities that may beset them. We need the dual fluid to admit a bounding surface.

Such bounded fluid configurations arise naturally for gauge theories with a deconfinement transition of first order. At temperatures just above the deconfinement transition, lumps of deconfined plasma, described at long wavelengths as fluids, can exist separated by a domain wall from the confining vacuum [12. A gravitational dual of a theory with this behavior is provided by ScherkSchwarz (SS) compactified $\mathrm{AdS}_{d+2}$ gravity, whose solutions asymptote to $\mathbb{M}^{d-1} \times \mathcal{S}^{1}$, with $\mathcal{S}^{1}$ standing for the distinguished SS circle. Under the approximation of a very thin domain wall, there are solutions for finite lumps of deconfined plasma whose dual gravitational solution must correspond to black objects localized near the infrared end of SS-AdS. These solutions are perfectly 
regular, provided that the SS circle shrinks to zero size in the bulk, in correspondence with the domain wall. This implies that the topology of the corresponding event horizon is given by the fibration of the SS circle $\mathcal{S}^{1}$ over the plasma lump geometry, with the circle shrinking to a point on the boundary. Plasma balls and plasma rings correspond, respectively, to spherical black holes and to black rings in the bulk. These were studied in [13] with an emphasis on the $\mathrm{AdS}_{5}$ case, and the full phase diagram for balls and rings in $\mathrm{AdS}_{6}$ was obtained more recently in [14]. Crucially, the horizon of the black hole is not mapped to the boundary of the fluid, but to its entire bulk. Then the apparent contradiction between minimal fluid surface area and maximal horizon area can be resolved.

Thus, this seems to be the correct framework to address the correspondence between black holes and lumps of fluid. One aim of this paper is to clarify the puzzles discussed above pertaining the variational principles that determine the equilibrium configurations of a fluid and of a black hole, as well as the relation between physical magnitudes in both cases.

Having dealt with this, we attempt to uncover in the physics of these plasma lumps some of the characteristic phenomena that higher-dimensional black holes exhibit (see e.g., [15, 16, 17]). In particular, we focus on plasma tubes, which are dual to black strings. As mentioned above, fluid analogues of the Gregory-Laflamme instability and of non-uniform black strings [18, 19] that branch off at its threshold have been investigated recently [1, 2, 3, 20, 21, 22. Here we reproduce and extend these results to include rotation, which uncovers novel phases of non-uniform tubes. But now we can regard these results as more than an analogy: following [7] they are actual solutions to a controlled approximation to the Einstein equations in SS-AdS and thus describe gravitational dynamics of black holes and black strings localized near the infrared in the SS-AdS spacetime1.

It turns out that these results show remarkable agreement with those found for black holes and black strings in asymptotically flat space. This prompts the question of whether the latter should admit, in some limit, a precise description in terms of fluid dynamics. After all, even if the equivalence between AdS black holes and fluids is very satisfying and promising, it does not yet explain the observations that suggested an analogy between asymptotically flat black holes and fluids in the first place. While we do not have a complete answer to this, we will make some observations that suggest that asymptotically flat black holes may be accurately described by fluid equations in the limit of very large number of dimensions.

The plan of the rest of the paper is the following: Section 2 reviews the basics of the relativistic hydrodynamics of plasma lumps, and provides the dual identification between temperature of the black hole and parameters at the surface of the fluid. Section 3 discusses the equivalence between several variational principles for fluids. This allows to understand how maximization of the entropy of a black hole can be equivalent to minimization of the surface area of a fluid. In section 4 we explore static and rotating configurations of plasmas in equilibrium in a space with one (large) compact direction, with a particular emphasis on tubes extended along this direction. In section 5, a detailed analysis of the Rayleigh-Plateau instability for these is performed. We obtain the dispersion relations for the unstable modes, both for static (Fig. 5) and spinning tubes (Fig. 6). After these

\footnotetext{
${ }^{1}$ Strictly speaking, the analysis of [7] refers to black branes dual to fluids that fill the spacetime they live in. We can reasonably expect that the extension to the situation of interest here should only be technically more complicated due to the absence of analytic solutions for plasma balls.
} 
analyses, we discuss in section 6 their range of validity, and in section 7 how they compare to the phases and stability of black strings in vacuum gravity. Section 8 revisits the issues posed at the beginning of the paper and suggests that vacuum black holes in the limit of large number of dimensions may admit a fluid description.

Note added: during the final stage of this project we have been informed of the work [23], which overlaps with some of our results.

\section{Hydrodynamic description of deconfined plasma lumps}

\subsection{Navier-Stokes and Young-Laplace equations}

Large black holes in AdS correspond to deconfined gluon plasma in the boundary CFT. In the longwavelength approximation, this plasma behaves as a fluid, whose properties can be deduced from its gravitational bulk description. In this approximation, one can perform a derivative expansion, whose leading order is given by a perfect fluid, while next orders introduce dissipative and diffusive phenomena. More precisely, it has been shown that the long-wavelength sector of pure AdS gravity is described by Navier-Stokes equations on the boundary, and the stress tensor of the corresponding fluid has been computed up to second order in the derivative expansion in five [7, 24] and four dimensions [25].

We are interested in the case where the boundary theory admits a confining phase, which close to the deconfinement temperature is separated from the deconfined plasma by a thin domain wall [12]. We assume that our plasma lumps are large enough to neglect the thickness of the wall. The analysis of this subsection follows largely [13. We shall neglect subleading dissipation and diffusion contribution.2. In this approximation, the deconfined plasma fluid behaves as a perfect fluid, and the leading order stress tensor is given by the sum of a perfect fluid part and a boundary surface contribution, describing the capillarity of the fluid,

$$
\begin{aligned}
& T^{\mu \nu}=T_{\text {perf }}^{\mu \nu}+T_{\text {bdry }}^{\mu \nu}, \\
& T_{\text {perf }}^{\mu \nu}=\left[(\rho+P) u^{\mu} u^{\nu}+P g^{\mu \nu}\right] \Theta(-f), \quad T_{\text {bdry }}^{\mu \nu}=-\sigma h^{\mu \nu}|\partial f| \delta(f) .
\end{aligned}
$$

Here, $u^{\mu}$ is the fluid velocity, $\rho, P$ and $\sigma$ are its density, pressure and surface tension respectively. The fluid boundary is defined by $f\left(x^{\mu}\right)=0$, has unit normal $n_{\mu}=\partial_{\mu} f /|\partial f|$ pointing towards the confining phase, and $h^{\mu \nu}=g^{\mu \nu}-n^{\mu} n^{\nu}$ is the projector onto the boundary. The velocity field $u^{\mu}$ is subject to the boundary condition

$$
u^{\mu} n_{\mu}=0,
$$

requiring that the fluid velocity must be orthogonal to the boundary normal or else the fluid would not be confined inside the boundary but flow through it. Finally, $\Theta(-f)$ is the Heaviside function $(\Theta(-f)=1$ inside the fluid and zero everywhere else).

\footnotetext{
${ }^{2}$ In particular, for stationary equilibrium solutions, these contributions vanish. We will comment on the effects of higher order corrections on the analysis of the perturbations near the end of the article.
} 
The equations describing the dynamics of the fluid follow from the conservation of the energy momentum tensor (2.1). The volume and boundary contributions must vanish independently; the former, projected along the fluid velocity $u_{\nu}$ yields the relativistic continuity equation,

$$
u^{\mu} \nabla_{\mu} \rho+(\rho+P) \nabla_{\mu} u^{\mu}=0
$$

(where $\nabla_{\mu} u^{\mu}=\theta$ is the expansion of the fluid) that we use to reduce the remaining equations to the relativistic Navier-Stokes equation,

$$
(\rho+P) u^{\mu} \nabla_{\mu} u^{\nu}=-\left(g^{\mu \nu}+u^{\mu} u^{\nu}\right) \nabla_{\mu} P .
$$

Here $u^{\mu} \nabla_{\mu} u^{\nu}=a^{\nu}$ is the acceleration of the fluid and $g^{\mu \nu}+u^{\mu} u^{\nu}$ is the projector onto the subspace orthogonal to $u^{\mu}$.

Now consider the boundary contribution to the conservation of the stress tensor,

$$
\left[(\rho+P) u^{\mu} u^{\nu}+P g^{\mu \nu}\right]|\partial f| n_{\mu}+\sigma \nabla_{\mu}\left(h^{\mu \nu}|\partial f|\right)=0 .
$$

Projecting this equation onto $n_{\nu}$, using (2.2), and integrating across the boundary we get

$$
P_{<}-P_{>}=\sigma K, \quad K \equiv h_{\mu}{ }^{\nu} \nabla_{\nu} n^{\mu},
$$

where $K$ is the trace of the boundary's extrinsic curvature (twice the mean curvature $H$ of the bounding surface), and $P_{<}-P_{>}$is the pressure jump when we cross the boundary from the exterior into the interior. Equation (2.6) is the Young-Laplace equation that describes the capillary pressure difference $\Delta P$ sustained across the interface boundary due to surface tension $\sigma$ [26].

\subsection{Stationary plasma configurations}

The Young-Laplace equation we found in the last subsection expresses the balance of forces on the plasma boundary and holds in the most general dynamical situation. We will turn now to the study of stationary plasma configurations in hydrodynamical and thermodynamical equilibrium.

To begin with, we need to characterize stationary configurations of plasma. Let us assume that the background spacetime is stationary, with stationarity timelike Killing vector $\xi$, and a set of commuting, linearly independent spacelike Killing vectors $\chi_{I}$, corresponding to the isometries of the background. Of the latter, the subset that commutes with the velocity field will also be symmetries of the fluid, $\mathcal{L}_{\chi_{I}} u=0$. The associated conserved charges will be the energy $E$ and the linear/angular momenta $J_{I}$ of the plasma associated to fluid symmetries.

Let $\mathcal{P}$ be the spatial region filled by the plasma, $\partial \mathcal{P}$ its boundary, and $u^{\mu}$ the velocity field of the fluid. Stationarity, for an isolated system, requires that there be no dissipation, and therefore the bulk and shear viscosity terms of the stress tensor must vanish. This condition is met if and

only if the expansion $\theta$ and the shear $\sigma^{\mu \nu}$ of the fluid both vanish. Under these assumptions, one can show that there exists a function $\alpha$ such that $\alpha u^{\mu}$ is a Killing vector.

Indeed, for a shearless, expansion-free velocity field,

$$
\nabla^{\mu} u^{\nu}=\omega^{\mu \nu}-u^{\mu} a^{\nu}
$$


where $\omega^{\mu \nu}$ is the vorticity of the fluid and $a^{\mu}$ its acceleration. Moreover, a fluid with local entropy density $s$ and local temperature $\mathcal{T}$ has to satisfy the Euler relation,

$$
\rho+P=\mathcal{T} s .
$$

Differentiating this equation and using the first law of thermodynamics, we get the Gibbs-Duhem relation $d P=s d \mathcal{T}$. Hence, since for a stationary configuration $u^{\mu} \nabla_{\mu} P=0$, the Navier-Stokes equations can be rewritten as

$$
a_{\mu}=-(\rho+P)^{-1} \nabla_{\mu} P=-\nabla_{\mu} \ln \mathcal{T},
$$

where, in the last step, we used both the Euler and the Gibbs-Duhem relations. Note that this equation expresses simply the fact that the heat flux vanishes

$$
q^{\mu}=-\kappa\left(g^{\mu \nu}+u^{\mu} u^{\nu}\right)\left(\nabla_{\nu} \mathcal{T}+a_{\nu} \mathcal{T}\right)=0
$$

or, in other words, stationary plasma configurations are both at hydrodynamical and thermal equilibrium. Substituting equation (2.9) in (2.7) it follows that

$$
\nabla_{(\mu}\left(\alpha u_{\nu)}\right)=\alpha u_{(\mu} \nabla_{\nu)} \ln (\alpha \mathcal{T})
$$

Hence, if we choose the function $\alpha$ to be inversely proportional to the local temperature of the plasma, $\alpha=T / \mathcal{T}$ with $T$ an integration constant, the vector field $\alpha u^{\mu}$ solves the Killing equations, and must therefore be a linear combination of the Killing vectors $\xi$ and $\chi_{\mathbb{l}} 3$. Hence, stationary configurations have a velocity field given by

$$
u=\frac{\mathcal{T}}{T}\left(\xi-\Omega_{I} \chi_{I}\right)
$$

The Killing vectors appearing here are a subset, although not necessarily the full set (since there may be rotational symmetries with no rotation velocity of the fluid in their direction), of the abelian symmetries of the fluid, which themselves are a subset of the symmetries of the background. The most general solution is determined by the constant parameters $T$ and $\Omega_{I}$, since the local temperature $\mathcal{T}$ is then fixed by the relation $u^{2}=-1$ to be

$$
\mathcal{T}=\gamma T
$$

where

$$
\gamma^{-1}=\sqrt{-\left(\xi-\Omega_{I} \chi_{I}\right)^{2}}
$$

is the redshift factor relating measurements done in the laboratory and comoving frames, and the constant $T$ is the equilibrium plasma temperature. It follows from the general form (2.12) of $u^{\mu}$ that a stationary equilibrium fluid configuration has to be in rigid roto-translational motion.

Combining the Euler relation (2.8) and the Young-Laplace equation (2.6), we can relate the temperature parameter $T$ of the fluid (which, for a non-boosted lump in an ultra-static background

\footnotetext{
${ }^{3}$ Actually, it could depend linearly on Killing vectors not commuting with $\chi_{I}$. However, one can always choose the set of Killing vectors $\chi_{I}$ to be adapted to the motion of the fluid under consideration.
} 
spacetime with $\xi^{2}=-1$, is the temperature of the fluid at the axis of rotation) to a combination of several magnitudes at the fluid surface,

$$
T=\frac{\sigma K+\rho}{\gamma s} .
$$

In the duality to a black hole, $T$ corresponds to the Hawking temperature of the horizon. We see that $T$ is not simply proportional to the surface tension or to the mean curvature, although it grows linearly with either of them. Note also that for a static fluid $K$ will be constant over the surface, but in a stationary configuration the curvature $K$ will adjust itself to variations of the fluid velocity near the boundary. Let us also mention that the angular velocities of the black hole horizon are identified with the $\Omega_{I}$.

So far we have not specified the equation of state of the fluid. We are interested in the $d$ dimensional (non-conformal) plasma describing the hydrodynamic limit of the $(d+1)$-dimensional CFT dual to Scherk-Schwarz reduced $\mathrm{AdS}_{d+2}$ gravity, whose equation of state in $d=n+3$ dimensions reads (see [13])

$$
\rho+P=\frac{n+4}{n+3}\left(\rho-\rho_{0}\right)
$$

and

$$
s=(n+4) \alpha^{1 /(n+4)}\left(\frac{\rho-\rho_{0}}{n+3}\right)^{\frac{n+3}{n+4}}, \quad \mathcal{T}=\left(\frac{\rho-\rho_{0}}{(n+3) \alpha}\right)^{\frac{1}{n+4}},
$$

with $\rho_{0}$ and $\alpha$ constants. This equation of state is normalized such that the vacuum pressure outside the plasma vanishes. When $n=0$, if we consider the theory that is dual to type II strings in $\mathrm{SS}_{-} \mathrm{AdS}_{5} \times S^{5}$, we have

$$
\alpha=\frac{\pi^{2} N^{2}}{8 T_{c}}, \quad \rho_{0}=\frac{\pi^{2} N^{2} T_{c}^{3}}{8} .
$$

The surface tension of the domain wall has been computed numerically in [12] for $n=0$ and $n=1$. In units of $\rho_{0} / T_{c}$ they obtain

$$
\sigma_{n=0}=2.0 \frac{\rho_{0}}{T_{c}}, \quad \text { and } \quad \sigma_{n=1}=1.7 \frac{\rho_{0}}{T_{c}} .
$$

For a plasma in equilibrium, it follows from (2.13) and (2.16) that the density and pressure satisfy the relations

$$
\rho=\rho_{*} \gamma^{n+4}+\rho_{0}, \quad P=\frac{\rho_{*}}{n+3} \gamma^{n+4}-\rho_{0},
$$

where

$$
\rho_{*}=(n+3) \alpha T^{n+4}
$$

is the difference $\rho-\rho_{0}$ between the plasma energy density and the energy density of the confining vacuum at points where $\gamma=1$. We see that all the dependence of the density on the position is entirely given by the $\gamma$ factors. 


\section{Variational principles for equilibrium plasma configurations}

In this section we will show that the Young-Laplace equation (2.6) can also be obtained using two equivalent variational principles, namely through a maximization of entropy and/or through a minimization of potential energy. As an easy byproduct we will see that these are also equivalent to minimization of the surface area for static fluids. Our formulation will be relativistic, fully covariant, and valid for a large class of stationary background spacetimes. While the relation between these two variational principles is probably well-known, we were not able to find it in the literature and we surmise that the relativistic covariant generalization that we present is original.

\subsection{Relativistic soap bubbles and second law of thermodynamics}

Equilibrium plasma configurations must have constant temperature and are expected to be those that extremize the entropy of the plasma while keeping its energy and momenta fixed. We will use this variational principle to give an alternative derivation the Young-Laplace equation, shedding a new light on the relation between black holes and their membrane analogues.

As explained in the previous section, equilibrium configurations on a stationary background spacetime are in rigid motion, and have a velocity field $u^{\mu}$ of the form (2.12), determined by the temperature $T$ of the plasma and the boost parameters $\Omega_{I}$. Again $\xi^{\mu}$ is the stationarity Killing vector and we choose a time coordinate $t$ such that $\xi=\partial_{t}$. This allows us to foliate the spacetime into constant $t$ hypersufaces $\Sigma_{t}$, that we use to define the conserved charges. We call $k^{\mu}$ the unit normal vector to these hypersurfaces, and we make the additional hypothesis that the Killing vectors $\chi_{I}$ generate isometries of $\Sigma_{t}$, i.e., $k \cdot \chi_{I}=0$. Then, given any Killing vector $\psi^{\mu}$, one can define the associated conserved charges

$$
\mathcal{Q}[\psi]=\int_{\Sigma_{t}} d v T_{\mu \nu} k^{\mu} \psi^{\nu}
$$

where $d v$ is the induced volume measure on $\Sigma_{t}$.

To perform the maximization of the entropy at constant energy and momenta, we define the action

$$
I[\mathcal{P}]=S[\mathcal{P}]-\beta E[\mathcal{P}]+\tilde{\omega}_{I} J_{I}[\mathcal{P}],
$$

where $\beta$ and $\tilde{\omega}_{I}$ are the Lagrange multipliers associated to our constraints. The total entropy of the fluid is the conserved charge associated to the entropy density current $s u^{\mu}$,

$$
S[\mathcal{P}]=-\int_{\mathcal{P}}(k \cdot u) s d v,
$$

while the energy and momenta are given by

$$
\begin{aligned}
& E[\mathcal{P}]=\int_{\mathcal{P}}[(\rho+P)(\xi \cdot u)(k \cdot u)+(k \cdot \xi) P] d v-\sigma \int_{\Sigma_{t}} k^{\mu} \xi^{\nu} h_{\mu \nu}|\partial f| \delta(f) d v, \\
& J_{I}[\mathcal{P}]=\int_{\mathcal{P}}(\rho+P)(k \cdot u)\left(\chi_{I} \cdot u\right) d v-\sigma \int_{\Sigma_{t}} h_{\mu \nu} k^{\mu} \chi_{I}^{\nu}|\partial f| \delta(f) d v .
\end{aligned}
$$

In addition to the bulk terms, both the energy $E$ and the momenta $J_{I}$ have a surface contribution

proportional to the surface tension. The boundary term in $E[\mathcal{P}]$ corresponds to the surface tension 
potential energy, and reduces to $\sigma$ times the area of the boundary of the fluid lump for a configuration respecting the spacetime symmetries in an ultra-static background. The boundary term in $J_{I}[\mathcal{P}]$ is present only if $\chi_{I} \cdot n \neq 0$ so the fluid boundary is not invariant under the action of $\chi_{I}$, for instance when the surface is not axisymmetric.

Taking the Lagrange multipliers to be given by

$$
\beta=1 / T, \quad \tilde{\omega}_{I}=\beta \Omega_{I},
$$

making use of the form (2.12) for $u$ and eliminating the entropy density $s$ by using the Euler relation, the action simplifies to

$$
I[\mathcal{P}]=-\beta \int_{\mathcal{P}}(k \cdot \xi) P d v+\beta \sigma \int_{\Sigma_{t}}(k \cdot \xi)|\partial f| \delta(f) d v .
$$

To extremize this action, we need to find its variation under a deformation of the region $\mathcal{P}$ occupied by the fluid. Notice that, since by hypothesis the configuration is stationary, the action is time independent, and its extrema are not changed if we integrate it over the time $t$. Then, noting that the spacetime volume element is

$$
d V=-(\xi \cdot k) d v d t
$$

it follows that the quantity we have to extremize assumes the form

$$
\int I[\mathcal{P}] d t=\beta \int_{\mathcal{M}} P \Theta(-f) d V-\beta \sigma \int_{\mathcal{M}}|\partial f| \delta(f) d V,
$$

where the integrals extend to the full spacetime $\mathcal{M}$, and the resulting functional is manifestly covariant. Also, note that the last integral is the area of the region of spacetime (world-volume) spanned by the boundary of the fluid. To extremize (3.8) under deformations of $\mathcal{P}$, we compute its variation under a change $\delta f$ of the function $f$ and integrate by parts the last term,

$$
\delta_{f} \int I[\mathcal{P}] d t=\beta \int_{\mathcal{M}}(P-\sigma K) \delta f \delta(f) d V .
$$

We used here the well-known fact that the variation of the area of an hypersurface is precisely given by twice the mean curvature, or its extrinsic curvature $K$. Therefore, requiring that (3.9) vanishes for any deformation $\delta f$ of the boundary, we obtain that the variational principle boils down to

$$
\sigma K=P
$$

where $P$ is the pressure jump at the boundary of the fluid. This, for non-vanishing external pressure, is again the Young-Laplace equation (2.6) that we derived in the last section using the conservation of the energy-momentum tensor. This time we found that it also follows from requiring extremization of plasma entropy for fixed energy and momenta. Note that the derivation assumes only the stationarity of the configuration and of the background geometry, and is independent of the fluid equation of state. Besides, the derivation does not assume any condition on the shape of $\mathcal{P}$, in particular it covers non-axisymmetric cases where the geometry of the fluid configuration does not share the symmetries of the fluid motion, as in the case of the two-lobed figures of equilibrium. 


\subsection{Equivalent variational principles for spinning lumps}

A universal behavior of fluids is that they always pick boundary configurations that reduce their potential energy for a fixed volume. For static solutions, this implies that the area of the fluid surface is minimized. For stationary solutions, the potential energy not only has a surface tension term but also a centrifugal contribution. We want to verify the equivalence between this potential energy minimization (hence a mechanical formulation) problem and the entropy maximization problem (a thermodynamic formulation) studied in the previous subsection.

To find an energetic formulation of the variational principle, we first observe that, using (2.12), the total action (3.2) assumes the form (3.6) in which the second term in the right hand side has the interpretation of surface potential energy, while the pressure integral has the interpretation of centrifugal potential energy, since the radial variation of pressure measures the centrifugal force per unit volume of fluid.

Therefore, we define a new action

$$
\widehat{I}[\mathcal{P}]=U_{\sigma}[\mathcal{P}]+U_{\mathrm{cf}}[\mathcal{P}]-\eta V[\mathcal{P}]
$$

where $U_{\sigma}[\mathcal{P}]=\sigma \mathcal{A}[\partial \mathcal{P}]$ is the surface tension potential energy associated to the area

$$
\mathcal{A}[\partial \mathcal{P}]=-\int_{\Sigma_{t}}(k \cdot \xi)|\partial f| \delta(f) d v
$$

the centrifugal potential energy, obtained by integrating the pressure over the plasma volume is

$$
U_{\mathrm{cf}}[\mathcal{P}]=\int_{\mathcal{P}}(k \cdot \xi)\left(P-P_{>}\right) d v,
$$

with $P_{>}$an integration constant, and finally the volume $V[\mathcal{P}]$ reads

$$
V[\mathcal{P}]=-\int_{\mathcal{P}}(k \cdot \xi) d v
$$

and is kept fixed as we perform the variation through the Lagrange multiplier $\eta$. Note that, in an ultra-static background, $\mathcal{A}[\partial \mathcal{P}]$ and $V[\mathcal{P}]$ reduce to the usual definitions of boundary area and volume of the plasma, respectively. Again, one can then easily verify that the Euler-Lagrange equation associated with (3.11) yields the Young-Laplace equation (2.6), and the Lagrange multiplier takes the value $\eta=P_{>}$, where $P_{>}$is the exterior vacuum pressure.

The two variational approaches, maximization of entropy and minimization of area, are equivalent because the actions (3.2) and (3.11) are proportional to each other. Indeed, starting with the action (3.2), successive use of (3.4), (3.5) and (2.8) yields the following relation between actions (3.2) and (3.11),

$$
I[\mathcal{P}]=-\beta \widehat{I}[\mathcal{P}]
$$

Since the two actions are the same up to a negative constant, minimizing the potential energy for fixed volume, $\delta \widehat{I}[\mathcal{P}]=0$, is naturally equivalent to maximizing the entropy for fixed conserved charges, $\delta I[\mathcal{P}]=0$. 
Finally note that in static configurations, $\Omega_{I}=0$, the pressure is constant inside the fluid and we can write

$$
I[\mathcal{P}]=-\beta \widehat{I}[\mathcal{P}]=-\beta \sigma(\mathcal{A}[\partial \mathcal{P}]-K V[\mathcal{P}])
$$

and when this action is extremized the Lagrange multiplier $K$ is required to be twice the mean curvature. Therefore, non-rotating equilibrium fluids have boundary shapes that minimize the surface area for fixed volume and their boundaries have constant mean curvature, a well known property of fluids.

Black holes satisfy the variational principle that their entropy is extremized for fixed energy and angular momenta - this is essentially the first law. In the duality between SS-AdS black holes and fluid lumps, the entropy, energy and spins are identified on both sides, while the temperature is mapped according to eqs. (2.15) and $\Omega_{I}$ are the angular velocities. Then our analysis shows that maximization of the black hole horizon area is equivalent, for static configurations, to minimization of the fluid surface area. When rotation is present, the connection is less simple geometrically, but still easily expressed as an extremization of a functional.

Thus, in this context the proposal in [1] to model black strings with non-gravitating cylinders of fluids can be made exact. There are, however, some differences between the analysis in refs. [1, 20] and ours: theirs was a study of non-relativistic fluids, whereas our fluids are relativistic. For static fluids the differences are small 4 , but for non-static or out of equilibrium situations the relativistic corrections become crucial. But the more important differences are that, first, we have a definite equation of state for the fluid and a specification of the value of the surface tension. This allows us to compute thermodynamic quantities of the fluid that are identified with those for the black hole, something that was impossible in [1, 20]. Second, the number of dimensions for the fluid and the black hole are different: a fluid in $d$ spacetime dimensions is mapped onto a black hole in $d+2$ dimensions. In particular, the entire volume of the fluid can be regarded as mapped onto the black hole horizon.

\section{Static and rotating equilibrium plasma configurations}

In this section we specialize to the study of stationary axisymmetric rigidly rotating plasma configurations in a flat spacetime and discuss the corresponding phase diagrams of solutions. These also represent the phase diagrams for the dual black objects. We start with a general description of the system we want to study, and then we obtain the differential equation for the shape of equilibrium plasmas. We find the static plasma configurations in any dimension $n$, and the rotating plasma configurations for $n=1$ and discuss the resulting phases.

\subsection{General formulation of the stationary and axisymmetric case}

The shape of stationary and axisymmetric plasma configurations is determined by the YoungLaplace equation. The background geometry is $d=n+3$ dimensional flat spacetime $\mathbb{R}_{t} \times \mathbb{R}^{n+1} \times S^{1}$

\footnotetext{
${ }^{4}$ Namely, $\rho+\mathcal{P} \sim \rho$ in the non-relativistic limit and the extrinsic curvature has time derivative terms that disappear in the non-relativistic limit. These time derivative terms are however vanishing when dealing with stationary solutions.
} 
(with $n \geq 1$ ), and we choose to work in coordinates such that the metric reads

$$
d s^{2}=-d t^{2}+d r^{2}+r^{2}\left(d \theta^{2}+\sin ^{2} \theta d \phi^{2}+\cos ^{2} \theta d \Omega_{n-2}^{2}\right)+d z^{2},
$$

where $\theta \in[0, \pi / 2], \phi \in[0,2 \pi)$ and $d \Omega_{n-2}^{2}$ is the metric of the unit $(n-2)$-sphere. For $n=1$ all the results can be reproduced by setting $\theta=\pi / 2$.

We consider rigidly rotating configurations with fluid velocity

$$
u^{\mu}=\gamma\left(\delta^{\mu}{ }_{t}+\omega_{\phi} \delta^{\mu}{ }_{\phi}\right), \quad \gamma=\left(1-g_{\phi \phi} \omega_{\phi}^{2}\right)^{-1 / 2},
$$

with constant angular velocity $\omega_{\phi}$. Note that a boost $\omega_{z}$ as well as rotation in other planes (if allowed by the dimension of the spacetime) could be easily added to our discussion.

These plasma configurations satisfy the equilibrium equation of state (2.20). Their entropy $S$ and conserved charges $E, J$ are given, respectively, by (3.3) and (3.4) with the relevant Killing vectors associated to (4.1) being $\xi=\partial_{t}$ and $\chi_{\phi}=\partial_{\phi}$. We will look for surfaces of revolution invariant under the action of these vectors.

\subsection{Static plasma lumps}

Our discussion will be very succint since these configurations were studied in [20] and we have rederived their main results. However, since we have an equation of state for the fluid we are able to discuss the properties of the solutions in a different manner: we display the phases in an entropy vs energy diagram. These quantities map directly into the entropy and energy of the dual black holes and black strings in SS-AdS. Therefore a direct comparison is possible to the diagrams that have been obtained for the black hole and black string phases in vacuum gravity.

For a profile of the form $r=R(z)$ the equations follow easily from the Young-Laplace equation (2.6) which, as we have discussed extensively, requires the mean curvature $K$ of the surface of a static fluid to be constant. The equilibrium equation of state (2.20) fixes this constant to

$$
K=\frac{\rho_{*}-(n+4) \rho_{0}}{(n+3) \sigma} .
$$

There are three families of solutions to this equation:

- Uniform plasma tubes (UT): These solutions have constant radius $r=R_{o}$ along the circle, so $K=n / R_{o}$. These are essentially configurations of a plasma ball in $d-1=n+2$ dimensions, times a straight line $z$, and have pressure and density

$$
P=n \frac{\sigma}{R_{o}}, \quad \rho=n(n+3) \frac{\sigma}{R_{o}}+(n+4) \rho_{0} .
$$

- Plasma balls $(B)$ : These solutions are characterized by $R(z)=\sqrt{R_{o}^{2}-z^{2}}$, i.e., they describe a $(n+1)$-dimensional sphere of radius $R_{o}$, and extrinsic curvature $K=(n+1) / R_{o}$, so

$$
P=(n+1) \frac{\sigma}{R_{o}}, \quad \rho=(n+1)(n+3) \frac{\sigma}{R_{o}}+(n+4) \rho_{0} .
$$



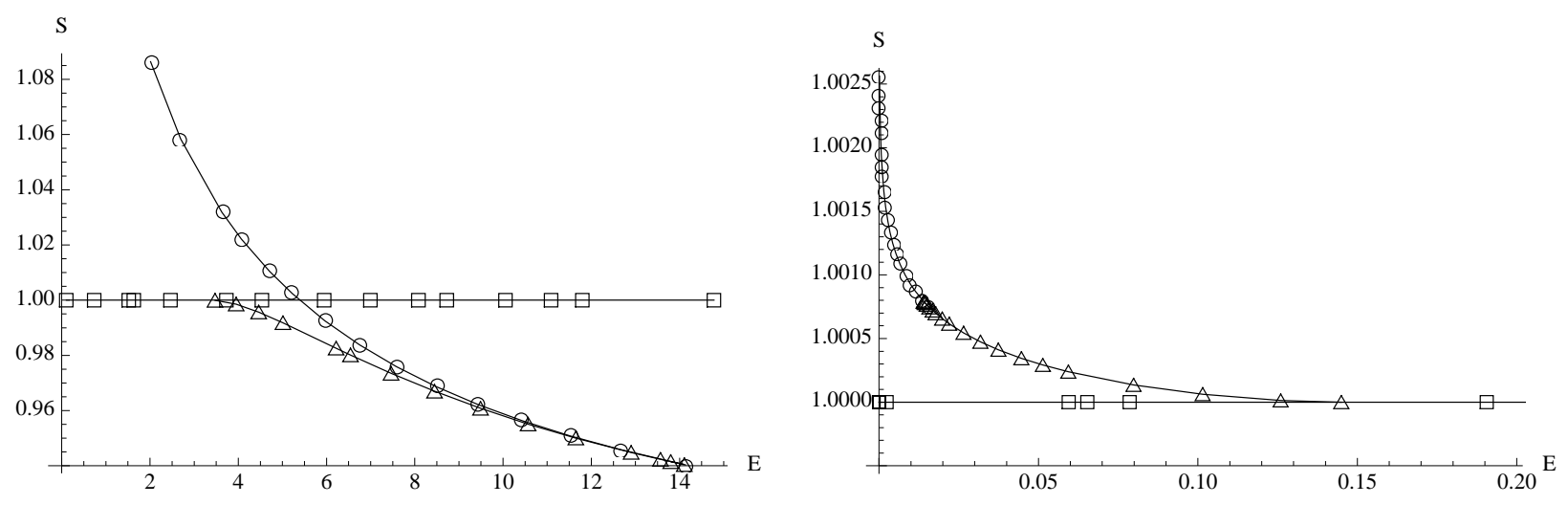

Figure 1: Entropy versus energy phase diagrams. The entropy is normalized to be one for uniform plasma tubes. Uniform plasma tubes, plasma balls and non-uniform plasma tubes correspond to squares, circles and triangles respectively. The left diagram, plotted for $n=1$, is representative for $n \leq 7$, and shows that the preferred configuration is the plasma ball for small energy, and the uniform plasma tube for higher energy. The unstable plasma ball branch merges with the non-uniform plasma tube branch in the merger point. Above the critical dimension, for $n \geq 8$ (the right diagram corresponds to $n=12$ ), the non-uniform plasma tubes become stable and become the preferred configuration for an intermediate range of energies. The left figure should be compared with Fig. 3 of [27] for the phases of non-uniform black strings. To plot these diagrams we used the values $\alpha=1, \rho_{0}=0$ and $\sigma=1$.

- Non-uniform plasma tubes (NUT): For $n \neq 0$, there is a third family of static equilibrium solutions that describe non-uniform plasma tubes. These configurations are found by solving numerically a first integral of the equation for the mean curvature.

We analyzed these solutions numerically, and constructed the corresponding phase diagrams in the $E-S$ plane. These are shown for $n=1$ (left) and $n=12$ (right) in Fig. 1. The preferred configuration, at a given energy, is given by the one with highest entropy. There is a critical dimension $n_{\star}=8$ for which the qualitative behavior changes. For $n \leq 7$, the entropically favored configuration is the plasma ball for small energy, which turns into a uniform plasma tube through a first order phase transition as the energy is increased. The non-uniform plasma tube branch has always a lower entropy (Fig. 1, left). However, for $n \geq 8$, the uniform plasma tubes benefit entropically from generating non-uniformities in an intermediate range of energies (Fig. 1, right).

The case $n=0$ is special, since the fluid lives in a two-dimensional space and the uniform plasma strips and plasma disks are the only solutions with constant mean curvature. Static plasma strip solutions have vanishing fluid pressure: since the section of the boundary at constant $z$ consists of just two points, there is no extrinsic curvature of the surface that must be balanced by pressure. This means that capillarity does not exert any force in the direction orthogonal to the plasma strip. This is consistent with the fact that the surface area does not change when the tube radius changes. In this case, the configuration is in a stable equilibrium, and no non-uniform plasma strip solution exists.

In the next section, we shall obtain a better understanding of these features in terms of the mechanical stability properties of the uniform plasma tubes. Indeed, the existence of the non- 
uniform plasma tube branch can be inferred from the fact that the uniform plasma tube changes its stability properties for a critical energy, at which it develops a static unstable mode. This critical unstable mode implies a bifurcation point to a new branch of static solutions that describe the non-uniform plasma tubes, and is associated to the Rayleigh-Plateau instability. For $n=0$, the corresponding threshold mode has infinite wavelength, so there cannot be any non-uniform branches connected to the uniform strip.

Note also that one can have an arbitrary number of coexisting disconnected plasma lumps. In the plasma fluid approximation, which is valid as long as they are far apart compared to the mean free path of the fluid, they do not feel each other 5 . In particular, other phases in which the fluid is distributed in multiple plasma balls can be present, but they have subleading entropy with respect to the single plasma ball in the phase diagram. Also, one can construct new branches of non-uniform plasma tubes, known as copy solutions, by unwrapping the original non-uniform plasma tube along the compact $z$ direction, and changing its periodicity. These new branches can be easily obtained through scaling arguments [28]. Since they are subleading in entropy we will not consider them.

\subsection{Rotating plasma lumps}

The problem of finding general equilibrium solutions in dimension $n$ is considerably complicated by the presence of rotation, which breaks the $S O(n+1)$ symmetry of the sections at constant $z$ to $S O(2) \times S O(n-1)$ and introduces a dependence on the angle $\theta$ in the profile of the lumps. Then one is forced to solve a partial differential equation. However, this problem is not present when $n=1$, where the transverse spheres are actually circles so there is the same symmetry as in the static case and the equations reduce to ODEs. Therefore we restrict our analysis of spinning lumps to $n=1$. Even if there may be phenomena of higher-dimensional tubes that we miss by this restriction, the case of $n=1$ still exhibits qualitatively new dynamics relative to the static situation.

We represent the boundary of the spinning lumps in terms of a height function $h(r) 6$

$$
f(r, z)=z-h(r)=0
$$

Then, use of the equilibrium equation of state (2.20) for the pressure and of the Young-Laplace equation (2.6) yields the ODE for a stationary axisymmetric plasma configuration,

$$
\frac{d}{d r}\left(\frac{r h^{\prime}}{\sqrt{1+h^{\prime 2}}}\right)+\frac{\rho_{*}}{4 \sigma} r\left(1-r^{2} \omega_{\phi}^{2}\right)^{-5 / 2}-\frac{\rho_{0}}{\sigma} r=0 .
$$

Some solutions to these equations were already studied in detail in [13, 14], namely, balls, pinched balls and rings. Now they are constrained to fit along the $z$ direction, which we are taking to be compact. Two other solutions, the rotating uniform tube and the uniform 'hollow tube' can be

\footnotetext{
${ }^{5}$ For the dual black holes, this means separations larger than the cosmological length scale $1 / \sqrt{-\Lambda}$, and their mutual gravitational interaction would involve massive Kaluza-Klein modes in the vacuum phase, which is subleading.

${ }^{6}$ For the uniform tubes, the simplest boundary parametrization is instead $f(r)=r-R_{o}=0$ and the Young-Laplace equation gives $\rho_{c}$ as a function of $\rho_{0}, R_{o}, \omega_{\phi}$.
} 
readily constructed out of them by simply taking the rotating plasma disk and the plasma annulus that appear in one less dimension and translating them uniformly along $z 7$ To make contact with this previous work we follow partially their notation and define the dimensionless variables,

$$
\widetilde{\omega}_{\phi}=\frac{\sigma \omega_{\phi}}{\rho_{0}}, \quad v=\omega_{\phi} r, \quad H(v)=\omega_{\phi} h(r), \quad k=\frac{1}{4} \frac{\rho_{*}}{\rho_{0}}, \quad \tilde{L}=\frac{\rho_{0}}{\sigma} L,
$$

where $L$ will be used to represent the length of the uniform tube with radius $R_{o}$ and $\tilde{L}$ the corresponding dimensionless quantity. Note also that reality of $\gamma=\left(1-v^{2}\right)^{-1 / 2}$ requires $0 \leq v \leq 1$.

Integrating twice (4.7) we get the function $H(v)$ that describes the profile of the spinning lumps,

$$
H(v)=\int_{v_{o}}^{v} \frac{-f(x)}{\left(g(x)^{2}-f(x)^{2}\right)^{\frac{1}{2}}} d x ; \quad \text { with } \quad f(v)=2 k-3\left(v^{2}+2 c\right) \gamma^{-3}, \quad g(v)=6 \widetilde{\omega}_{\phi} v \gamma^{-3} .
$$

where $c$ is an integration constant. Plasma rings have also a inner surface: its profile is described by a similar expression obtained by multiplying the first relation in (4.9) by -1 and replacing $v_{o} \rightarrow v_{\mathrm{i}}$ (with $v_{\mathrm{i}}$ being the inner velocity; for details see [14]).

To make contact again with [14] we define the dimensionless energy, angular momentum and entropy as

$$
\widetilde{E}=\frac{\rho_{0}^{2} E}{4 \pi \sigma^{3}}, \quad \widetilde{J}=\frac{\rho_{0}^{3} J}{20 \pi \sigma^{4}}, \quad \widetilde{S}=\frac{\rho_{0}^{\frac{11}{5}} S}{20 \pi \alpha^{\frac{1}{5}} \sigma^{3}},
$$

where $E, J, S$ follow from (3.4) and (3.3). It is also useful to introduce the following functions:

$$
\begin{aligned}
\widetilde{E}_{t}\left(v_{o}\right) & =\frac{\widetilde{L}}{12 \widetilde{\omega}_{\phi}^{2}}\left(2 k \gamma_{o}^{5}\left(v_{o}^{2}+2-2 \gamma_{o}^{-5}\right)+3 v_{o}\left(v_{o}+\widetilde{\omega}_{\phi}\right)\right) \\
\widetilde{J}_{t}\left(v_{o}\right) & =\frac{\widetilde{L} k \gamma_{o}^{5}}{30 \widetilde{\omega}_{\phi}^{3}}\left(2 \gamma_{o}^{-5}-2+5 v_{o}^{2}\right), \quad \widetilde{S}_{t}\left(v_{o}\right)=\frac{\widetilde{L} k^{4 / 5}}{6 \widetilde{\omega}_{\phi}^{2}}\left(\gamma_{o}^{3}-1\right) .
\end{aligned}
$$

and

$$
\begin{aligned}
& \widetilde{E}_{b}(a, b)=\frac{1}{\widetilde{\omega}_{\phi}^{3}} \int_{a}^{b} d v\left(v H(v)\left(k\left(4+v^{2}\right) \gamma^{7}+1\right)+\widetilde{\omega}_{\phi} v \sqrt{1+H^{\prime}(v)^{2}}\right) \\
& \widetilde{J}_{b}(a, b)=\frac{k}{\widetilde{\omega}_{\phi}^{4}} \int_{a}^{b} v^{3} \gamma^{7} H(v) d v, \quad \widetilde{S}_{b}(a, b)=\frac{k^{4 / 5}}{\widetilde{\omega}_{\phi}^{3}} \int_{a}^{b} v \gamma^{5} H(v) d v
\end{aligned}
$$

We now discuss the seven families of axisymmetric spinning plasma lumps that we found to be solutions of (4.7) (we present the ones with non-trivial profiles in Figs. 2, 3, and 4. The figures should be rotated along the vertical axis $v=0$ ):

- Uniform plasma tubes (UT): These have constant radius $R_{o}$ along their length $L$. They have $\widetilde{\omega}_{\phi}=v_{o}\left(k \gamma_{o}^{5}-1\right)$, where $\left.\gamma_{o} \equiv \gamma\right|_{v_{o}}$. Their energy, angular momentum and entropy are $\widetilde{E}=\widetilde{E}_{t}\left(v_{o}\right), \widetilde{J}=\widetilde{J}_{t}\left(v_{o}\right)$ and $\widetilde{S}=\widetilde{S}_{t}\left(v_{o}\right)$ as defined in (4.11).

\footnotetext{
${ }^{7}$ Observe that this is not possible for pinched balls since they do not exist for $n=0$.
} 

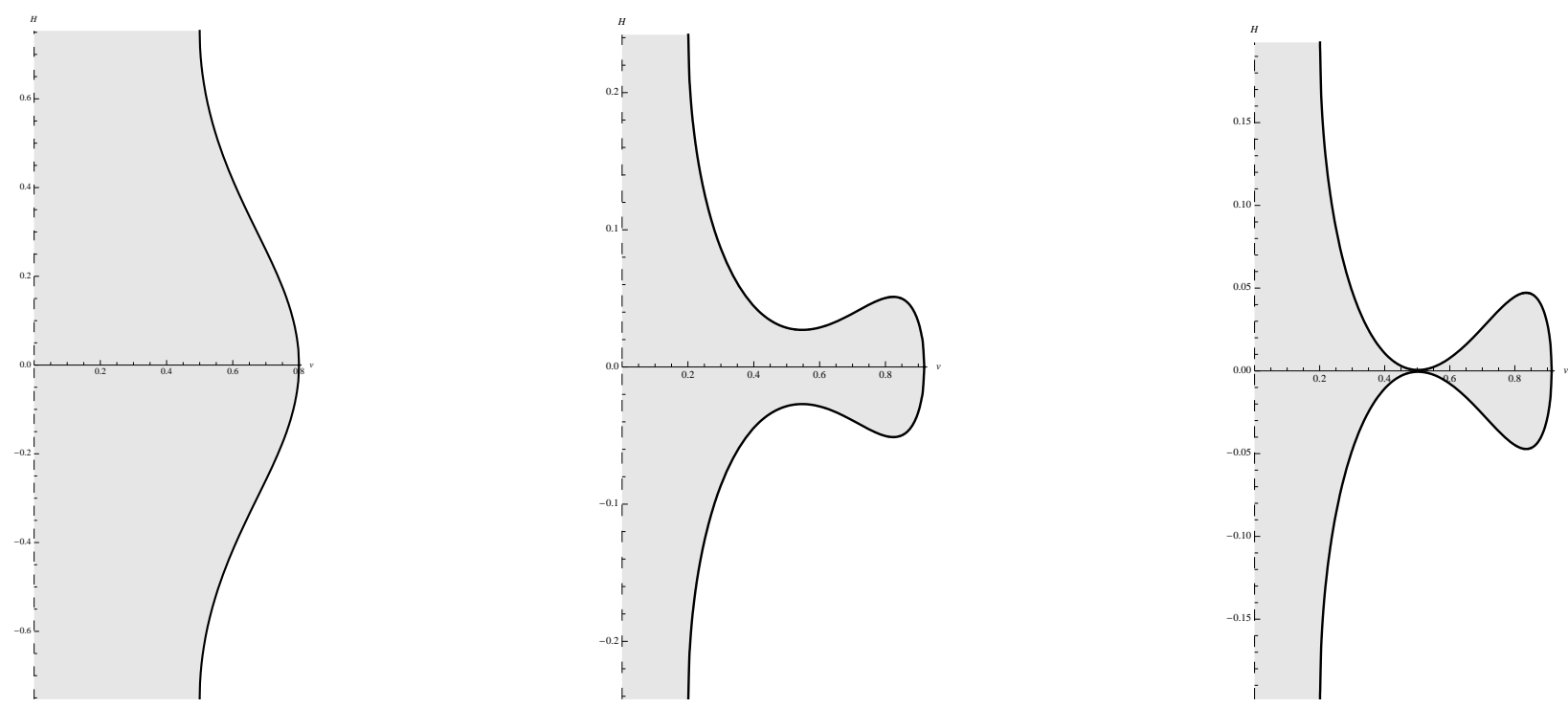

Figure 2: Figures of equilibrium of a rotating plasma tube. From left to right, an ordinary non-uniform rotating plasma tube $\left(v_{m}=.5, v_{o}=.8, k=.628024\right)$, a pinched plasma tube $\left(v_{m}=.201016, v_{o}=.918\right.$, $k=.15)$ and a plasma tube on the verge of splitting off a plasma ring $\left(v_{m}=.201016, v_{o}=.9133, k=.15\right)$. The parameters have been chosen such that the three tubes share the same periodicity.

- Uniform hollow tubes (UHT): These have two constant radii, outer $R_{o}$ and inner $R_{\mathrm{i}}$, along their length $L$ delimiting the tubular region inside which the fluid lies. The Young-Laplace equation on the inner and outer boundaries fixes a constraint between these two radii, which can be written as $\widetilde{\omega}_{\phi}=v_{o}\left(k \gamma_{o}^{5}-1\right)=v_{\mathrm{i}}\left(1-k \gamma_{\mathrm{i}}^{5}\right)$, where $\left.\gamma_{o, \mathrm{i}} \equiv \gamma\right|_{v_{o, \mathrm{i}}}$. The energy, angular momentum and entropy are $\widetilde{E}=\widetilde{E}_{t}\left(v_{o}\right)-\widetilde{E}_{t}\left(v_{\mathrm{i}}\right), \widetilde{J}=\widetilde{J}_{t}\left(v_{o}\right)-\widetilde{J}_{t}\left(v_{\mathrm{i}}\right)$ and $\widetilde{S}=\widetilde{S}_{t}\left(v_{o}\right)-\widetilde{S}_{t}\left(v_{\mathrm{i}}\right)$, with the definitions in (4.11).

- Non-uniform plasma tubes (NUT): These tubes have a radius that varies from a non-vanishing minimum, $v_{m}$, up to a maximum, $v_{o}$, as we go along their length. At both these extreme points we have $H^{\prime}(v)=-\infty$, and in between them $H^{\prime}(v)$ is always negative (so the point in between the two extreme points where $H^{\prime \prime}(v)$ changes sign has $\left.H^{\prime}(v)<0\right)$; see Fig. 2a. In the critical NUT configuration, $H^{\prime}(v)=0$ at the inflection point and the NUT and pinched NUT (discussed next) meet in what we call the critical NUT. These boundary conditions imply the following relations between the parameters,

$$
\begin{aligned}
c & =\frac{k}{3}\left(1-v_{m}^{2}\right)^{-3 / 2}-\frac{1}{2} v_{m}^{2}-\widetilde{\omega}_{\phi} v_{m} \\
\widetilde{\omega}_{\phi} & =\frac{1}{6 v_{o}}\left(1-\frac{v_{m}}{v_{o}}\right)^{-1}\left[2 k\left(\left(1-v_{o}^{2}\right)^{-3 / 2}-\left(1-v_{m}^{2}\right)^{-3 / 2}\right)-3\left(v_{o}^{2}-v_{m}^{2}\right)\right] .
\end{aligned}
$$

The energy, angular momentum and entropy of the NUTs are given, respectively, by $\widetilde{E}=$ $\widetilde{E}_{t}\left(v_{m}\right)+\widetilde{E}_{b}\left(v_{m}, v_{o}\right), \widetilde{J}=\widetilde{J}_{t}\left(v_{m}\right)+\widetilde{J}_{b}\left(v_{m}, v_{o}\right)$ and $\widetilde{S}=\widetilde{S}_{t}\left(v_{m}\right)+\widetilde{E}_{b}\left(v_{m}, v_{o}\right)$ where we use the functions defined in (4.11) and (4.12). 

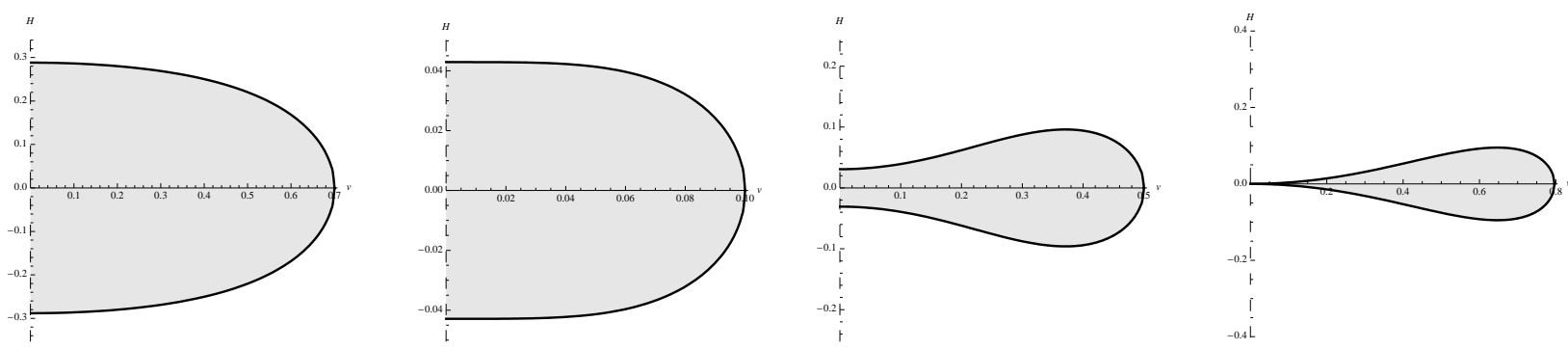

Figure 3: Figures of equilibrium of a rotating plasma ball. From left to right, an ordinary rotating drop $\left(v_{o}=.7, k=2\right)$, a marginal rotating drop $\left(v_{o}=.1, k=21\right)$, a pinched plasma ball $\left(v_{o}=.5, k=.83\right)$ and a pinched ball on the verge of splitting of a plasma ring $\left(v_{o}=.7965, k=.5\right)$.
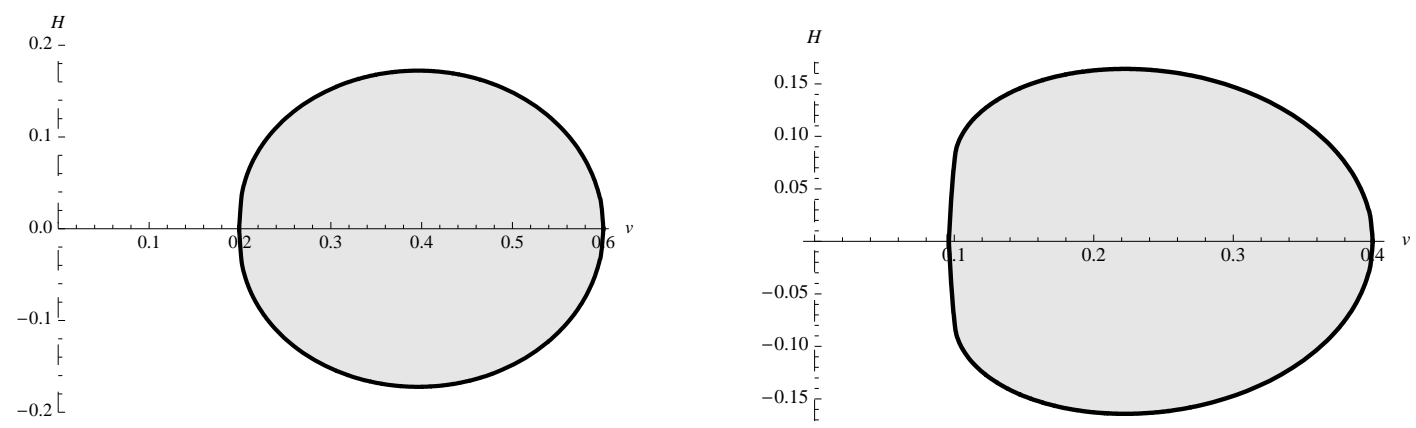

Figure 4: Figures of equilibrium of a rotating plasma ring. On the left, a thin plasma ring $\left(v_{\mathrm{i}}=.2, v_{o}=.6\right.$, $k=9)$ and on the right a fat plasma ring $\left(v_{\mathrm{i}}=.1, v_{o}=.4, k=2\right)$.

- Pinched non-uniform plasma tubes ( $p N U T$ ): These tubes also have a radius that varies from a non-vanishing minimum, $v_{m}$, up to a maximum, $v_{o}$. At both these extreme points we have $H^{\prime}(v)=-\infty$. But, contrary to the NUT solutions, $H^{\prime}(v)$ now changes sign twice as $v$ runs from $v_{m}$ to $v_{o}$ (so the point in between the two extreme points where $H^{\prime \prime}(v)$ changes sign has now $H^{\prime}(v)>0$ ), see Fig. 2, b. These boundary conditions still imply the relations (4.13) and the conserved charges of the pinched NUTs are given by the same functional defined for the NUT in the previous item. As the rotation increases we reach a configuration where we have a pNUT and a plasma tube on the verge of splitting off a plasma ring; see Fig. 2.c.

- Plasma balls (B): These plasma configurations are oblate spheres. At the extreme points they satisfy $H^{\prime}(v=0)=0$ and $H^{\prime}\left(v=v_{o}\right)=-\infty$. Moreover, $H^{\prime}(v)$ is always negative at the boundary as we go from $v=0$ up to $v=v_{o}$; see Fig. 3. The condition $H^{\prime}(0)=0$ fixes $c=k / 3$ and $H^{\prime}\left(v_{o}\right)=-\infty$ requires $f\left(v_{o}\right)=g\left(v_{o}\right)$ which fixes $\widetilde{\omega}_{\phi}=\frac{1}{6 v_{o}}\left(2 k \gamma_{o}^{3}-2 k-3 v_{o}^{2}\right)$. Finally, the condition $H^{\prime}(v)<0$ in the interval $\left(0, v_{o}\right)$ implies $k>1$. For $k=1$ the critical ball configuration is reached (see Fig. 3. b). Here, the plasma ball joins the pinched ball branch discussed next. The conserved charges are given, respectively, by $\widetilde{E}=\widetilde{E}_{b}\left(0, v_{o}\right), \widetilde{J}=\widetilde{J}_{b}\left(0, v_{o}\right)$ and $\widetilde{S}=\widetilde{E}_{b}\left(0, v_{o}\right)$ where we use the functions defined in (4.12). The balls must have a distance between their poles smaller than the compact dimension length. The same applies to the solutions we discuss next. 
- Pinched plasma balls $(p B)$ : These balls also have $H^{\prime}(0)=0$ and $H^{\prime}\left(v_{o}\right)=-\infty$ which again requires $c=k / 3$ and $\widetilde{\omega}_{\phi}=\frac{1}{6 v_{o}}\left(2 k \gamma_{o}^{3}-2 k-3 v_{o}^{2}\right)$. But contrary to the plasma balls, $H^{\prime}(v)$ changes from positive to negative sign in the interval $\left(0, v_{o}\right)$; see Fig. 3.c. This requires $0<k<1$. However not all $k$ in this interval give a physical solution. They must further satisfy $H(v=0)>0$. At the critical situation where $H(0)=0$ the pinched plasma ball branch joins the fat plasma ring phase discussed next (see Fig. 3, d). The conserved charges of the pinched balls are given by the same expressions as for the balls.

- Plasma rings $(R)$ : These annulus configurations are characterized by $H^{\prime}\left(v_{\mathrm{i}}\right)=+\infty$ and $H^{\prime}\left(v_{o}\right)=-\infty$ at the inner and outer boundary, respectively, and for having $H\left(v_{\mathrm{i}}\right)=H\left(v_{o}\right)=$ 0 . This requires that $f\left(v_{\mathrm{i}}\right)=-g\left(v_{\mathrm{i}}\right)$ and $f\left(v_{o}\right)=g\left(v_{o}\right)$. For fixed outer boundary velocity, $v_{o}=\omega_{\phi} R_{o}$, there are two possible inner velocities $v_{\mathrm{i}}$ satisfying these conditions. The smaller one describes the fat plasma ring (that joins the pinched ball branch at the critical configuration where $v_{\mathrm{i}} \rightarrow 0$ ) and the larger $v_{\mathrm{i}}$ describes the thin ring (see Fig. 44). The two rings meet at a regular critical ring when the two aforementioned $v_{\mathrm{i}}$ 's are equal. The energy, angular momentum and entropy of the rings are given, respectively, by $\widetilde{E}=\widetilde{E}_{b}\left(v_{\mathrm{i}}, v_{o}\right), \widetilde{J}=\widetilde{J}_{b}\left(v_{\mathrm{i}}, v_{o}\right)$ and $\widetilde{S}=\widetilde{E}_{b}\left(v_{\mathrm{i}}, v_{o}\right)$ with functions defined in (4.12).

There also appears the possibility of non-uniform hollow tubes (NUHT), i.e., hollow tubes with an inhomogeneous profile along $z$, but we have not obtained direct evidence for them. If they exist, it would be interesting to increase their rotation and investigate their possible pinches and connections to other phases.

Having identified these families of plasma solutions, it should be interesting to represent their properties in a phase diagram where we fix the periodic direction length and the energy of the solution, and let the entropy vary with the angular momentum (alternatively, we could also fix $\widetilde{L}$

and $\widetilde{J}$ and represent the solutions in the $\widetilde{S}(\widetilde{E})$ diagram). This requires substantial and delicate numerical work since this is a shooting problem on three parameters. The curves for uniform tubes and balls with a fixed conserved charge can be obtained relatively easily but the numerical search of the non-uniform tubes is considerably more difficult. We leave this for future work.

\section{$5 \quad$ Rayleigh-Plateau instability of plasma tubes}

Non-relativistic fluid jets and liquid bridges in between two disk plates are unstable against the so-called Rayleigh-Plateau instability if their length is larger than their transverse perimeter. This instability is active even in the absence of gravity and surface tension is the crucial mechanism responsible for it: in the simplest case, capillarity forces conspire to reduce the surface area of the fluid. This is a long wavelength instability that makes the fluid cylinder pinch-off.

In this section we show that uniform plasma tubes are naturally afflicted by the relativistic Rayleigh-Plateau instability and we will study in detail its properties. We start by getting a good understanding of the mechanism responsible for the instability noting that there are perturbation modes that decrease the plasma tube potential energy while keeping its volume fixed (so this statement is independent of whether or not the fluid is compressible, i.e., of the equation of state). Equivalently, in view of the results of section 3, these modes are unstable because they increase the 
entropy of the system while keeping its conserved charges fixed. In subsection 5.1, this approach will allow to find the minimum length below which the uniform tube can be stable. Then in subsection 5.2 we will find the dispersion relation for this instability.

In the dual gravitational system, uniform black strings are expected to suffer from the gravitational Gregory-Laflamme instability. It is also a long wavelength instability that makes black strings longer than the horizon radius pinch. We will see that the plasma surface tension and gravitational instabilities have similar properties.

\subsection{Unstable threshold mode from entropy or potential energy variation}

\subsubsection{Threshold mode for static and boosted plasmas}

Consider an axisymmetric perturbation on a uniform plasma tube,

$$
r(z)=R_{o}+\epsilon R_{1} \cos (k z)+\epsilon^{2} R_{2}, \quad \epsilon \ll 1 .
$$

We take the tube length to be given by the Rayleigh-Plateau wavelength because that is where it might break, $L=\lambda=2 \pi / k$. The volume of the perturbed tube, up to a second order expansion, is then

$$
V=\lambda \frac{\pi^{(n+1) / 2}}{\Gamma\left(\frac{n+3}{2}\right)} R_{o}^{n-1}\left(R_{o}^{2}+\frac{n+1}{2}\left(\frac{n}{2} R_{1}^{2}+2 R_{o} R_{2}\right) \epsilon^{2}\right)+\mathcal{O}\left(\epsilon^{3}\right) .
$$

We require that the volume of the tube is kept fixed up to second order in the perturbation (equivalently, its conserved charges must be kept fixed) so this constrains $R_{2}$ as a function of the lower order radius,

$$
R_{2}=-\frac{n}{4} \frac{R_{1}^{2}}{R_{o}}
$$

The difference between the perturbed and unperturbed surface tension potential energy is

$$
\Delta U_{\sigma}=\sigma \frac{2 \pi^{(n+1) / 2}}{4 \Gamma\left(\frac{n+1}{2}\right)}\left(k^{2} R_{o}^{2}-n\right) R_{1}^{2} \epsilon^{2}+\mathcal{O}\left(\epsilon^{3}\right)
$$

where we used (5.3). Unstable modes are those that decrease the potential energy, i.e., surface area, of the fluid tube. So the condition $\Delta U_{\sigma} \leqslant 0$ for fixed volume requires

$$
k R_{o} \leqslant \sqrt{n}
$$

For static plasmas the threshold unstable mode is then $k=\sqrt{n} / R_{o}$ and it increases with the spatial dimension. Finally, a boost along the $z$-direction has only a kinematical effect on this instability, Lorentz-contracting the threshold wavelength [29].

\subsubsection{Threshold mode for rotating plasma and non-axisymmetric modes}

We now generalize the previous study to rotating and boosted uniform plasma tubes, as well as non-axisymmetric perturbations on it. Again, we will restrict to the $n=1$ dimensional case, since for higher $n$, the profile of the drop has a polar angle dependence that prevents an analytical study. 
The general perturbation on the uniform plasma tube reads,

$$
r(z, \phi)=R_{o}+\epsilon R_{1} \cos (k z) \cos (m \phi)+\epsilon^{2} R_{2}, \quad \epsilon \ll 1 .
$$

This perturbation includes axisymmetric modes $(m=0)$ as well as non-axisymmetric ones (integer $m \neq 0$ ). Again, we take the tube length to be equal to the instability wavelength, $L=\lambda=2 \pi / k$.

The volume of the perturbed tube, up to a second order expansion, is

$$
V=\lambda \pi R_{o}^{2}+\frac{\lambda}{4}\left(\pi\left(R_{1}^{2}+8 R_{o} R_{2}\right)+R_{1}^{2} \frac{\sin (4 m \pi)}{4 m}\right) \epsilon^{2}+\mathcal{O}\left(\epsilon^{3}\right) .
$$

We require that the volume of the tube is kept fixed up to second order in the perturbation (equivalently, its conserved charges must be kept fixed) so this constrains $R_{2}$ as a function of the lower order radius,

$$
R_{2}=-\frac{R_{1}^{2}}{4 R_{o}}, \quad \text { for } m=0 ; \quad R_{2}=-\frac{R_{1}^{2}}{8 R_{o}}, \quad \text { for } m \neq 0 .
$$

The potential energy of the tube is the sum of the surface tension and centrifugal contributions as defined in section 3. Using the constraint (5.8), the total potential energy difference between the perturbed and unperturbed configurations $\Delta U=\Delta U_{\sigma}+\Delta U_{\mathrm{cf}}$ is then given by $\left(\delta_{m}=1\right.$ if $m=0$ and vanishes otherwise)

$$
\Delta U=\frac{\pi \lambda \sigma}{4 R_{o}}\left(1+\delta_{m}\right)\left(\left(k^{2} R_{o}^{2}-1+m^{2}\right)-\frac{5}{4} \frac{\rho_{*} \omega_{\phi}^{2} R_{o}^{3}}{\sigma}\left(1-R_{o}^{2} \omega_{\phi}^{2}-\omega_{z}^{2}\right)^{-7 / 2}\right) R_{1}^{2} \epsilon^{2}+\mathcal{O}\left(\epsilon^{3}\right) .
$$

The Rayleigh-Plateau instability is active for wavenumbers that decrease the potential energy of the fluid tube. So the condition $\Delta U \leqslant 0$ for fixed volume requires

$$
k^{2} R_{o}^{2}+m^{2} \leqslant 1+\frac{5}{4} \frac{\rho_{*} \omega_{\phi}^{2} R_{o}^{3}}{\sigma}\left(1-R_{o}^{2} \omega_{\phi}^{2}-\omega_{z}^{2}\right)^{-7 / 2}
$$

The inverse of the ratio $\rho_{*} \omega_{\phi}^{2} R_{o}^{3} / \sigma$, that measures the competition between the surface tension and centrifugal effects, is often called the rotating Bond number or Hocking parameter. Rotation increases the critical wavenumber making the plasma tube unstable for a wider range of wavelengths. Only axisymmetric modes are unstable in the static case but when rotation is added, non-axisymmetric modes can become also unstable for sufficiently high velocity. We will discuss the dual gravitational interpretation of these unstable modes in section 7

\subsection{Rayleigh-Plateau dispersion relation}

In this subsection we want to address the stability of a uniform plasma tube when we perturb it. The dynamics of the perturbations is dictated by the hydrodynamic equations, subject to appropriate boundary conditions.

Perturbations take the plasma away from thermal equilibrium and therefore viscosity and diffusion effects start to contribute. The energy-momentum tensor of the fluid includes now not only the perfect fluid and the boundary surface tension terms (2.1), but also a dissipative contribution. As 
we will show, the uniform plasma tube is afflicted by the Rayleigh-Plateau instability and surface tension is the mechanism responsible for it. Viscosity and diffusion play no role on the activation of the instability and have a subleading effect on the dispersion relation, simply correcting the threshold wavelength and the time-scale of the instability. Hence, in our analysis we shall neglect the dissipation contribution to the fluid energy-momentum tensor, and simply comment at the end the subleading effects it introduces.

To study the stability of rigidly rotating uniform plasma tubes, we consider a generic unperturbed uniform tube with velocity (4.2). The precise expression for the unperturbed plasma pressure and density is fixed once we specify the equation of state. In particular, for the plasma we are interested in, these are specified by equations (2.16) and (2.20). The particular choice of the equation of state will however not be fundamental in our analysis.

A generic perturbation on the uniform tube is described as

$$
u^{\mu}=u_{(0)}^{\mu}+\delta u^{\mu}, \quad P=P_{(0)}+\delta P, \quad \rho=\rho_{(0)}+\delta \rho,
$$

where we denote an unperturbed quantity by $Q_{(0)}$, and the perturbation as $\delta Q$. The perturbed state (5.11) must satisfy the relativistic continuity and Navier-Stokes equations, (2.3) and (2.4). The Young-Laplace equation (2.6) provides then a boundary condition for the perturbed problem. After eliminating the $0^{\text {th }}$ order terms using the unperturbed hydrodynamic equations, the continuity and the Navier-Stokes equations yield, up to first order in the perturbation,

$$
\begin{gathered}
u_{(0)}^{\mu} \partial_{\mu} \delta \rho+\delta u^{\mu} \partial_{\mu} \rho_{(0)}+\left(\rho_{(0)}+P_{(0)}\right) \nabla_{\mu} \delta u^{\mu}+(\delta \rho+\delta P) \nabla_{\mu} u_{(0)}^{\mu}=0 \\
\left(\rho_{(0)}+P_{(0)}\right)\left(\delta u^{\mu} \nabla_{\mu} u_{(0)}^{\nu}+u_{(0)}^{\mu} \nabla_{\mu} \delta u^{\nu}\right)+(\delta \rho+\delta P) u_{(0)}^{\mu} \nabla_{\mu} u_{(0)}^{\nu} \\
+\left(g^{\mu \nu}+u_{(0)}^{\mu} u_{(0)}^{\nu}\right) \nabla_{\mu} \delta P+\left(\delta u^{\nu} u_{(0)}^{\mu}+u_{(0)}^{\nu} \delta u^{\mu}\right) \nabla_{\mu} P_{(0)}=0 .
\end{gathered}
$$

The density and pressure perturbations are not independent. They are related by the equation of state (2.16), valid also out of equilibrium. In particular, perturbation of the first relation in (2.16) yields

$$
\delta \rho=(n+3) \delta P .
$$

Since we can expand any perturbation in a Fourier series, we restrict the analysis to a generic mode and consider a perturbation that disturbs the boundary surface according to

$$
r=R(t, z, \phi), \quad R(t, z, \phi)=R_{o}+\epsilon e^{\omega t} e^{i k z+i m \phi}, \quad \epsilon \ll R_{o},
$$

where $R_{o}$ is the unperturbed radius of the uniform tube (note that for the rotating $n>1$ case it is $\theta$-dependent). Positive $\omega$ describes an instability with wavenumber $k$. These modes break axisymmetry if $m \neq 0$. The unit normal of (5.15) is

$$
n_{\mu}=|n|^{-1}\left(-R_{t}^{\prime} \delta_{\mu}^{t}+\delta_{\mu}^{r}-R_{\phi}^{\prime} \delta_{\mu}^{\phi}-R_{z}^{\prime} \delta_{\mu}^{z}\right), \quad|n|=\left(1-R_{t}^{\prime 2}+\frac{R_{\phi}^{\prime 2}}{r^{2}}+R_{z}^{\prime 2}\right)^{1 / 2} .
$$

Naturally, we look for perturbations of the fluid quantities that have the same form as the boundary disturbance,

$$
\delta Q(t, r, z, \phi)=\delta Q(r) e^{\omega t} e^{i k z+i m \phi}, \quad \delta Q \equiv\left\{\delta u^{\mu}, \delta P, \delta \rho\right\}
$$


The perturbed hydrodynamic equations (5.12) and (5.13) must be supplemented by appropriate boundary conditions. The first one demands normal stress balance on the boundary. This means that the pressure perturbation that solves (5.12) and (5.13) must also satisfy the third perturbed hydrodynamic equation, namely the equation that follows from perturbing the Young-Laplace equation (2.6),

$$
\mathrm{BC} \mathrm{I}:\left.\quad \delta P\right|_{\mathrm{bdry}} \simeq \sigma\left[\left.K\right|_{R(t, z, \phi)}-\left.K\right|_{R_{o}}\right]-\left[\left.\left(P_{<}^{(0)}-P_{>}^{(0)}\right)\right|_{R(t, z, \phi)}-\left.\left(P_{<}^{(0)}-P_{>}^{(0)}\right)\right|_{R_{o}}\right],
$$

where on the rhs we evaluate the expression at the perturbed boundary $r=R(t, z, \phi)$ defined in (5.15) and subtract the unperturbed contribution evaluated at $r=R_{o}$. For our plasma, $P_{<}^{(0)}-P_{>}^{(0)}$ is obtained from (2.20), and the extrinsic curvature is given from its definition (2.6) using the unit normal (5.16).

The second boundary condition is a kinematic condition requiring that the normal component of the fluid velocity on the boundary satisfies the perturbed version of (2.2),$u_{(0)}^{\mu} \delta n_{\mu}+\delta u^{\mu} n_{\mu}^{(0)}=0$, where $\left.\delta n_{\mu} \equiv n_{\mu}\right|_{R(t, z, \phi)}-n_{\mu}^{(0)}$ and the unperturbed normal is $\left.n_{\mu}^{(0)} \equiv n_{\mu}\right|_{R_{o}}=\delta_{\mu}^{r}$. This ensures that the velocity perturbation leaves the fluid confined inside the boundary. This boundary condition then reads

$$
\mathrm{BC} \text { II : }\left.\quad \delta u^{r}\right|_{\mathrm{bdry}} \simeq\left(1-R_{o}^{2} \omega_{\phi}^{2}-\omega_{z}^{2}\right)^{-\frac{1}{2}}\left(\omega+i m \omega_{\phi}+i k \omega_{z}\right) \epsilon e^{\omega t} e^{i k z+i m \phi} .
$$

We have now all the ingredients needed to find perturbations of the form (5.15) and (5.17) that might develop an instability on the tube. We will find that there is indeed a long wavelength instability known in non-relativistic systems as the Rayleigh-Plateau instability (see for example [30]). It afflicts our plasma tube when, roughly, its length is larger than its transverse radius. We will first analyze this instability and its dispersion relation $\omega(k)$ for a static uniform tube in any dimension $n$. This is the simplest case where the crucial ingredients necessary to activate the instability are present. Then we will study the changes introduced in the instability properties by rotation and boost, as well as the effects of non-axisymmetric modes. This will be done in the $n=1$ case, where the analysis can be done analytically, but we expect the results to hold qualitatively also for $n>1$. Finally, we will discuss briefly the subleading effects that viscosity would introduce in the dispersion relation.

\subsubsection{Perturbations of static tubes in any dimension}

Consider a static uniform plasma tube with unperturbed radius $R_{o}$ and $u_{(0)}^{\mu}=\delta^{\mu}$, in $(n+3)$ flat spacetime dimensions, and deform its boundary by axisymmetric perturbations $(m=0)$ of the form (5.15). The perturbed continuity and Navier-Stokes equations, (5.12) and (5.13) have unstable modes that correspond to the Rayleigh-Plateau instability, as we show in the sequel. Solving (5.13) with perturbations (5.17), we find that the non-vanishing $\delta u^{\mu}$ are (at higher order other components will arise)

$$
\delta u^{r}(r)=-\omega^{-1}\left(\rho_{(0)}+P_{(0)}\right)^{-1} \frac{d \delta P(r)}{d r}, \quad \delta u^{z}(r)=-i \frac{k}{\omega}\left(\rho_{(0)}+P_{(0)}\right)^{-1} \delta P(r),
$$


which, replaced in the continuity equation (5.12), give

$$
\frac{d^{2} \delta P(r)}{d r^{2}}+\frac{n}{r} \frac{d \delta P(r)}{d r}-p^{2} \delta P(r)=0, \quad p=k\left(1+(n+3) \frac{\omega^{2}}{k^{2}}\right)^{\frac{1}{2}}
$$

The $\omega$ term in the definition of $p$ arises from the first term in (5.12) proportional to $\delta \rho$ and use of the equation of state (5.14). Equation (5.21) is a modified Bessel equation, whose solutions are the modified Bessel functions of the first kind $I_{ \pm \frac{n-1}{2}}(p r)$ and second kind $K_{\frac{n-1}{2}}(p r)$. Of these, $I_{-\frac{n-1}{2}}(p r)$ and $K_{\frac{n-1}{2}}(p r)$ diverge as $r^{-\frac{n-1}{2}}$ as $r \rightarrow 0$ and we discard them. Therefore, the regular solution of (5.21) at the origin is

$$
\begin{aligned}
& \delta P(r)=A r^{-\frac{n-1}{2}} I_{\frac{n-1}{2}}(p r), \\
& \delta u^{r}(r)=-\frac{A}{\omega\left(\rho_{(0)}+P_{(0)}\right)} r^{-\frac{n-1}{2}}\left(p I_{\frac{n-1}{2}}^{\prime}(p r)-\frac{n-1}{2 r} I_{\frac{n-1}{2}}(p r)\right), \\
& \delta u^{z}(r)=-\frac{i k A}{\omega\left(\rho_{(0)}+P_{(0)}\right)} r^{-\frac{n-1}{2}} I_{\frac{n-1}{2}}(p r),
\end{aligned}
$$

where we defined $I_{\nu}^{\prime}(x) \equiv \partial_{x} I_{\nu}(x)$, and where $A$ is a constant that is fixed by the boundary condition (5.18) to the leading-order value

$$
A \simeq \epsilon \sigma \frac{R_{o}^{\frac{n-1}{2}-2}}{I_{\frac{n-1}{2}}\left(p R_{o}\right)}\left(k^{2} R_{o}^{2}+\omega^{2} R_{o}^{2}-n\right) .
$$

With this knowledge, and using the relations $I_{\nu}^{\prime}(x)=I_{\nu+1}(x)+\frac{\nu}{x} I_{\nu}(x)$ and (2.20), the boundary condition (5.19) yields the desired dispersion relation $\omega=\omega(k)$, which reads

$$
\omega^{2}=\frac{n+3}{n+4} \frac{\sigma}{\rho_{*} R_{o}^{3}} \frac{p R_{o} I_{\frac{n+1}{2}}\left(p R_{o}\right)}{I_{\frac{n-1}{2}}\left(p R_{o}\right)}\left(n-k^{2} R_{o}^{2}-\omega^{2} R_{o}^{2}\right), \quad p=k\left(1+(n+3) \frac{\omega^{2}}{k^{2}}\right)^{\frac{1}{2}} .
$$

A plot of this dispersion relation $\omega(k)$ for several values of the dimension $n$ is shown in Fig. 5. To obtain these plots we have needed to specify the value of $\frac{\sigma}{\rho_{*} R_{o}}$. This amounts to considering plasma tubes of a specific width $R_{o}$ relative to the length scale $\sigma / \rho_{*}$, which is essentially the mean free path of the plasma. In principle we should vary this width and study the dispersion relation as a function of it. The shape of the dispersion relation is indeed modified as $\frac{\sigma}{\rho_{*} R_{o}}$ grows to values of order one. However, the validity of the fluid description requires that we consider small $\frac{\sigma}{\rho_{*} R_{o}}$ (see section 66). It turns out that in this case the qualitative aspects of the dispersion relation hardly change for different values of $\frac{\sigma}{\rho_{*} R_{o}}$ and the shapes shown in Fig. 5 are generic. For definiteness, we have taken

$$
\frac{\sigma}{\rho_{*} R_{o}}=\frac{12}{n+6} 10^{-6}
$$

so as to produce the plot. But we insist that the dimension-dependence in this choice, and hence in the curves in the plot, is completely arbitrary: it only amounts to choosing tubes of a particular width in each dimension. 


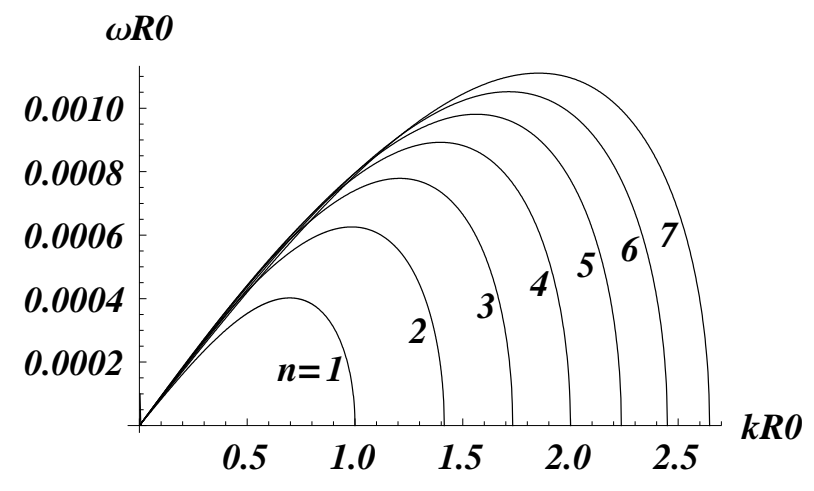

Figure 5: Plot of the dimensionless dispersion relation $\omega(k)$ for the Rayleigh-Plateau instability in a static uniform tube for several spacetime dimensions $d=n+3$. The instability strength and threshold wavenumber increase as the spacetime dimension grows. Intuitively, this is because the "same" perturbation decreases more the surface area of the plasma boundary (increases more the plasma entropy) as the dimension increases. Note also that the most unstable mode satisfies $\left.\omega R_{o}\right|_{\max } \gg \frac{\sigma}{\rho_{0} R_{o}}$, and is thus within the regime of validity of the hydrodynamic description (see section [6). This plot is to be qualitatively compared with Fig. 3 of $[5]$ for the GL instability.

The Rayleigh-Plateau instability is active when $\omega>0$. The instability gets stronger as the spacetime dimension increases since the threshold wavenumber, as well as the wavenumber and frequency where the maximum instability occurs, increase when $n$ grows.

There are two special points that we can read easily from (5.24). One is the point where the curves cross $\omega=0$, which gives the threshold wavenumber below which the Rayleigh-Plateau instability is active: $k R_{o}=\sqrt{n}$. So unstable modes satisfy condition (5.5), confirming the threshold mode that we obtained previously from entropical or energetical arguments in subsection 5.1 . The second point is at $k=0$, and one can expand the Bessel functions in (5.24) to check that $\omega$ at $k=0$ always vanishes, independently of $\frac{\sigma}{\rho_{*} R_{o}}$.

\subsubsection{Rotating and boosted plasma lumps}

We want to consider now how rotation and boost of the fluid modify the dispersion relation, and study the effect of non-axisymmetric modes $(m \neq 0)$. We shall restrict to the $n=1$ case where an analytical approach is viable. Take a uniform plasma tube rigidly rotating with velocity (4.2) and with unperturbed constant radius $R_{0}$. The unperturbed state satisfies, once the equation of state for the fluid is introduced, equations (2.16) and (2.20) with $n=1$.

Disturb now the plasma boundary with perturbations of the form (5.15). To get the velocity perturbations we have to solve (5.13) assuming perturbations of the form (5.17). One finds, after use of (5.14), (in (5.26)-(5.28) we restore the velocity of light factor $c$ to make the perturbative 
expansion that we do next more clear)

$$
\begin{aligned}
\delta u^{t}(r) & =\frac{\omega_{\phi} r^{2}}{c^{2}} \delta u^{\phi}(r), \\
\delta u^{r}(r)= & -\frac{4}{5 \rho_{*}} \frac{\varpi \gamma^{-1}}{\varpi^{2}+4 \omega_{\phi}^{2} \chi}\left[\partial_{r} \delta P(r)+\left(\frac{2 i m}{r} \frac{\omega_{\phi}}{\varpi}-3 \gamma^{2} r \frac{\omega_{\phi}^{2}}{c^{2}}\right) \delta P(r)\right], \\
\delta u^{\phi}(r)= & \frac{4}{5 \rho_{*}} \frac{\gamma^{-1}}{\varpi^{2}+4 \omega_{\phi}^{2} \chi}\left[\frac{2 \omega_{\phi} \chi}{r} \partial_{r} \delta P(r)-\frac{i m \varpi}{r^{2}} \delta P(r)-\frac{\omega_{\phi} \gamma^{2}}{c^{2}}\left(\varpi^{2}+10 \omega_{\phi}^{2} \chi\right) \delta P(r)\right], \\
\delta u^{z}(r)= & -i \frac{4}{5 \rho_{*} \gamma} \frac{k}{\varpi} \delta P(r)-\frac{4 \gamma}{5 \rho_{*} c^{2}}\left[\omega_{z} \delta P\right. \\
& \left.\quad-\frac{2 \omega_{\phi}^{2} \omega_{z} r}{\varpi^{2}+4 \omega_{\phi}^{2} \chi}\left(\partial_{r} \delta P(r)+\frac{2 i m \varpi}{r} \frac{\omega_{\phi}}{\varpi} \delta P(r)-\frac{3 \omega_{\phi}^{2} \gamma^{2} r}{c^{2}} \delta P(r)\right)\right],
\end{aligned}
$$

where we defined

$$
\varpi \equiv \omega+i m \omega_{\phi}+i k \omega_{z}, \quad \chi \equiv 1+\frac{\omega_{\phi}^{2} \gamma^{2} r^{2}}{c^{2}} .
$$

Note that (5.26) satisfies the perturbed version of $u^{\mu} u_{\mu}=-c^{2}$, namely $u_{\mu}^{(0)} \delta u^{\mu}=0$. Equations (5.26) can now be replaced in the continuity equation,

$$
\frac{d \delta u^{r}}{d r}+\frac{1}{r} \delta u^{r}+i m \delta u^{\phi}+i k \delta u^{z}+\omega \delta u^{t}+4 \gamma^{2} r \frac{\omega_{\phi}^{2}}{c^{2}} \delta u^{r}+\frac{16 \varpi \gamma}{5 \rho_{*} c^{2}} \delta P=0 .
$$

As will become clear, it is not possible to get an analytical expression for the dispersion relation unless we work in the small velocity regime, $\omega_{\phi} R_{o}, \omega_{z} \ll c$. Since the Rayleigh-Plateau instability is already present in the static case and we just want to find if adding velocity to the solution increases or decreases the instability strength and threshold unstable mode, we will solve the hydrodynamic equations in this regime (we will further represent higher order terms of the velocity generically by $\left.\mathcal{O}\left(\omega_{i}\right)\right)$. This restriction is also justified by the fact that the regime of validity of the hydrodynamic description and thus of our results is restricted to small rotation rates, as we will argue in section 6. So, to leading order (5.26) reduces to

$$
\begin{aligned}
& \delta u^{t}(r)=\mathcal{O}\left(\omega_{i}^{2}\right), \\
& \delta u^{r}(r)=-\frac{4}{5 \rho_{*}} \frac{\varpi}{\varpi^{2}+4 \omega_{\phi}^{2}}\left(\partial_{r} \delta P(r)+\frac{\omega_{\phi}}{\varpi} \frac{2 i m}{r} \delta P(r)\right)+\mathcal{O}\left(\omega_{i}^{2}\right), \\
& \delta u^{\phi}(r)=\frac{4}{5 \rho_{*}} \frac{\varpi}{\varpi^{2}+4 \omega_{\phi}^{2}} \frac{1}{r}\left(\frac{2 \omega_{\phi}}{\varpi} \partial_{r} \delta P(r)-\frac{i m}{r} \delta P(r)\right)+\mathcal{O}\left(\omega_{i}^{2}\right), \\
& \delta u^{z}(r)=-i \frac{4}{5 \rho_{*}} \frac{k}{\varpi} \delta P(r)+\mathcal{O}\left(\omega_{i}^{2}\right),
\end{aligned}
$$

The perturbed continuity equation yields then to leading order in the velocity 8

$$
\frac{d^{2} \delta P(r)}{d r^{2}}+\frac{1}{r} \frac{d \delta P(r)}{d r}-\left[\eta^{2}+\frac{m^{2}}{r^{2}}\right] \delta P(r)+\mathcal{O}\left(\omega_{i}^{2}\right)=0, \quad \eta \equiv k\left(1+\frac{4 \omega^{2}}{k^{2}}\right)^{\frac{1}{2}}\left(1+\frac{4 \omega_{\phi}^{2}}{\varpi^{2}}\right)^{1 / 2} .
$$

\footnotetext{
${ }^{8}$ Notice that the last term in (5.28) has a contribution $\omega / c^{2}$ that we keep because our expansion is only on the velocities but not on the frequency. This contribution is responsible for the $\omega^{2} / k^{2}$ term in $\eta$.
} 
This is again a modified Bessel equation with solutions $I_{m}(\eta r)$ and $K_{m}(\eta r)$ (note that if we had kept higher order terms we would not be able to solve analytically the differential equation). We discard the $K_{m}(\eta r)$ solution that diverges at the origin and thus obtain the regular solution

$$
\begin{aligned}
& \delta P(r) \simeq A I_{m}(\eta r), \\
& \delta u^{r}(r)=-\frac{4 A}{5 \rho_{*}} \frac{\varpi \eta}{\varpi^{2}+4 \omega_{\phi}^{2}}\left(I_{m}^{\prime}(\eta r)-\frac{2 i m}{\eta r} \frac{\omega_{\phi}}{\varpi} I_{m}(\eta r)\right)+\mathcal{O}\left(\omega_{i}\right) .
\end{aligned}
$$

The final expression for $\delta u^{\phi}, \delta u^{z}$ (that we do not need) can then be obtained from (5.29).

The boundary condition (5.18) fixes the ratio $A / \epsilon$ to leading order as

$$
\frac{A}{\epsilon} \simeq \frac{\sigma R_{o}^{-2}}{I_{m}\left(\eta R_{o}\right)}\left[k^{2} R_{o}^{2}+\omega^{2} R_{o}^{2}-1+m^{2}-\frac{5}{4} \frac{\rho_{*} \omega_{\phi}^{2} R_{o}^{3}}{\sigma}\left(1-\omega_{\phi}^{2} R_{o}^{2}-\omega_{z}^{2}\right)^{-7 / 2}\right] .
$$

Use of this ratio on the boundary condition (5.19) yields finally the leading dispersion relation $\omega(k)$

$$
\begin{aligned}
\varpi^{2} \simeq-4 \omega_{\phi}^{2}+ & \frac{4 \sigma}{5 \rho_{*} R_{o}^{3}}\left[\frac{\eta R_{o} I_{m+1}\left(\eta R_{o}\right)}{I_{m}\left(\eta R_{o}\right)}+m\left(1-i \frac{2 \omega_{\phi}}{\varpi}\right)\right] \\
& \times\left(1-m^{2}+\frac{5}{4} \frac{\rho_{*} \omega_{\phi}^{2} R_{o}^{3}}{\sigma}\left(1-\omega_{\phi}^{2} R_{o}^{2}-\omega_{z}^{2}\right)^{-7 / 2}-\omega^{2} R_{o}^{2}-k^{2} R_{o}^{2}\right) .
\end{aligned}
$$

A plot of this dispersion relation $\omega(k)$ for different values of $\omega_{\phi}$ (and $\omega_{z}=m=0$ ) is shown in Fig. 6. The dispersion relation (5.33), valid in the small velocity regime, is expect to be a good approximation up to velocities of the order $v_{o} \sim 0.1-0.5$. The Rayleigh-Plateau instability is active when $\omega>0$. The marginal unstable mode with $\omega=0$ satisfies condition (5.10), confirming the threshold mode obtained in subsection 5.1.2. As the rotation $\omega_{\phi}$ is increased, the threshold

wavenumber, as well as the wavenumber and frequency where the maximum instability occurs, and the instability grows stronger. Fixing $\omega_{\phi} \neq 0$, increasing $\omega_{z}$ also makes the instability stronger. Finally note that contrary to the static case where only axisymmetric modes were unstable, now non-axisymmetric modes can also become unstable for sufficiently high rotation.

Note that we decided to keep the higher order terms in velocities in the boundary condition (5.32) and in (5.33) instead of doing a Taylor expansion. The reason being that from the entropic/energetic computation we get the threshold condition (5.10) in the marginal unstable case. The computation leading to this condition is exact to all orders in the velocity, so we do not expand (5.32) that is responsible for the unstable wavenumber cut-off in (5.33).

In section 6 we will discuss the regime of validity of the hydrodynamic description. Our results are valid for $\left\{\omega R_{o}, k R_{o}\right\} \gg \frac{\sigma}{\rho_{0} R_{o}}$. There is a wide range of parameters for which this condition is satisfied. In particular, the physically relevant most unstable mode of Figs 5 and 6 fits very well in this regime. Near the extreme points of the dispersion relation where $\omega=0$ the results must be read with caution.

\subsubsection{Viscosity}

We have neglected in our analysis the effects of viscosity, which is absent from stationary solutions but should play a role in time-dependent processes such as the Rayleigh-Plateau instability. 


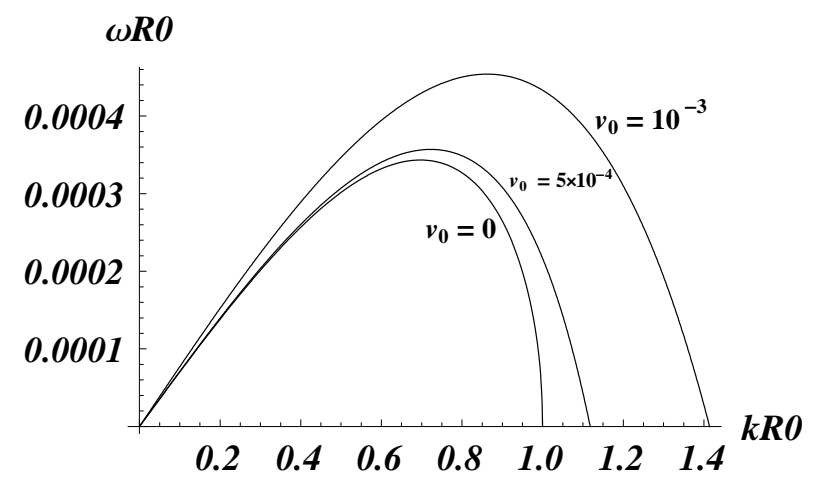

Figure 6: Plot of the dimensionless dispersion relation $\omega(k)$ for the Rayleigh-Plateau instability in a rotating uniform tube for dimensionless angular velocity $v_{o}=0 ; 5 \times 10^{-4} ; 10^{-3}$, where $v_{o} \equiv \omega_{\phi} R_{o}$. The instability strength and threshold wavenumber increase as the rotation grows. Intuitively, this is because the centrifugal force scales with the radial distance and thus it is bigger in a crest than in a trsough of the instability. The numerical data corresponds to take $\frac{4 \sigma}{5 \rho_{*} R_{o}}=10^{-6}, \omega_{z}=0$ and $m=0$. The dispersion relation (5.33), valid in the small velocity regime, is expect to be a good approximation up to velocities of the order $v_{o} \sim 0.1-0.5$. Note also that the most unstable mode satisfies $\left.\omega R_{o}\right|_{\max } \gg \frac{\sigma}{\rho_{0} R_{o}}$, and is thus within the regime of validity of the hydrodynamic description (see section 66). This plot should be compared with Fig. 1 of [40] for the GL instability of rotating black strings.

These effects are well understood in the the non-relativistic Rayleigh-Plateau instability, which has been the object of exhaustive theoretical, numerical and experimental study. It does not seem unreasonable to assume that the effect of viscosity in the relativistic and non-relativistic instabilities is qualitatively similar.

Viscosity plays a subleading role in the activation of the RP instability, although the instability can get considerably weaker if the viscosity is very high. Generically, it increases the wavelength of the most unstable mode, and weakens the strength of the instability [30]. Viscosity has been experimentally seen to play an important role at later stages in the time evolution of the instability, when the fluid tube pinches off, as we discuss in sec. 7

Dissipation increases considerably the technical challenge of solving numerically the hydrodynamic perturbed equations. This is because without it, these are ODEs with boundary conditions, while when dissipation is present we must deal with a coupled system of second order differential equations for the pressure and velocity perturbations, which cannot be studied analytically and requires advanced numerical treatment.

\section{Regime of validity}

We now discuss the limits of validity of the different approximations we have made in our derivations in the previous sections.

First of all, for the fluid description of the deconfined plasma to be accurate the thermodynamic quantities must vary slowly over the mean free path $\ell_{\text {mfp }}$ of the fluid, which is of the order of the mass gap of the theory, or equivalently of the order of the deconfinement temperature. That is, all 
length scales $\lambda$ in the fluid must be

$$
\lambda \gg T_{c}^{-1} \sim \frac{\sigma}{\rho_{0}} .
$$

For the same reason, thermal fluctuations must remain small. A good estimate for the valid regime is obtained when the maximum fractional rate of change of the fluid local temperature is small

$$
\left.\left.\frac{\delta \mathcal{T}}{T_{c}}\right|_{\max } \sim \partial_{r} \ln \gamma\right|_{\max } \ll 1
$$

This occurs for

$$
\frac{\omega_{\phi}^{2} R_{o}^{2}}{1-\omega_{\phi}^{2} R_{o}^{2}} \ll \frac{\rho_{o} R_{o}}{\sigma} .
$$

For plasma balls and rings this condition is satisfied for a wide range of lumps as long as we are away from extremality where the temperature vanishes [13. For plasma tubes the angular velocity $\omega_{\phi}$ increases with $R_{o}$ so the above condition is fulfilled as long as the tubes do not rotate too fast. This does not affect our analysis in section 5.2 .2 since, in order to have analytic control on the equations, we only considered the regime of small rotation. This condition also imposes restrictions on the validity of the Rayleigh-Plateau instability analysis. To guarantee that the thermodynamic quantities of the fluid vary on a length scale small when compared with the mean free path we must demand that the Rayleigh-Plateau unstable frequencies and wavenumbers satisfy

$$
\left\{\omega R_{o}, k R_{o}\right\} \gg \frac{\sigma}{\rho_{0} R_{o}} .
$$

Since the most unstable mode dominates the instability we must guarantee that this condition is satisfied in the vicinity of the maximum of the dispersion relation. We find that for $\frac{\sigma}{\rho_{0} R_{o}} \lesssim 10^{-4}$ this condition is satisfied, and things get better as $\frac{\sigma}{\rho_{0} R_{o}}$ becomes smaller. Recall that for the plots of Figs. 5 and 6 we took $\frac{\sigma}{\rho_{0} R_{o}} \sim 10^{-6}$, well within the validity range.

Second, the interface between the confined and deconfined phases, i.e., the fluid surface, has a finite thickness of the order $1 / T_{c}$ [12], and therefore the delta-like surface approximation we used is valid provided that the curvature of the surface is small with respect to $1 / T_{c}$. So, for plasma balls and rings the analysis is valid when the boundary radii $R_{\mathrm{o}}, R_{\mathrm{i}}$, and $R_{\mathrm{o}}-R_{\mathrm{i}}$ are large compared to $T_{c}^{-1}$. This is the case if the plasma energy is large and away from the extremal configurations [13. For plasma tubes the requirements are, analogously, $R_{\mathrm{o}}, R_{m}$, and $R_{\mathrm{o}}-R_{m}$ much larger than $T_{c}^{-1}$.

Finally, we neglected the dependence of the surface tension on the temperature and other thermodynamic quantities of the fluid. For consistency we must then demand that on the boundary between the confined and deconfined phases the temperature of the plasma must remain everywhere close to the critical temperature $T_{c}$. The values of $\mathcal{T}$ and $T_{c}$ at the boundary surfaces remain close for a large range of energies and angular momenta (see Fig. 9 of [13]), both for balls and rings as well as for plasma tubes, always away from the extremal limits.

\section{Rayleigh-Plateau and Gregory-Laflamme}

The dynamics of lumps of fluid that we have analyzed should admit, via the reasoning in [7, a mapping to the dynamics of black holes near the infrared bottom in SS-AdS, in the limit in 
which the black hole size is much larger than the thickness of the domain wall that connects it to the confining 'AdS soliton' vacuum. In this case, the correspondence between black holes and lumps of fluid can be established and the solutions of the fluid equations can be regarded as actual gravitational calculations in a controlled perturbative (in boundary derivatives) expansion to the Einstein equations in AdS, reformulated in an appropriate way.

This implies that our results for the dynamics of plasma tubes in $d$ dimensions can be regarded as describing gravitational physics of black strings in SS-AdS ${ }_{d+2}$ spacetime. However, such black string solutions have not been constructed directly yet and therefore a comparison cannot be made between results obtained using dual-fluid techniques and more conventional gravitational analyses.

Nevertheless, it is remarkable that the dynamics of fluid tubes from our analysis is strikingly similar to what is known for black strings in asymptotically flat space, for which a fairly large amount of results are available. It has been pointed out that all the different plasma ball, pinched ball and plasma ring phases found in [13, 14] in $d$ dimensions have black hole, pinched black hole, and black ring analogues in asymptotically flat $d+2$-dimensional gravity [31, 32, 33].

This parallelism extends to most aspects of the solutions we have discussed. Ref. [20] already observed that the phase diagrams for static plasma lumps (shown here in Fig. 1) resemble the corresponding phase diagrams for black strings and black holes localized in a Kaluza-Klein circle [34, 16]. Let us add that if we compare our Fig. 1]a with Fig. 3 of [27], the two figures can be strikingly superposed. Note that in Fig. 3 of [27] the gravitational merger between the non-uniform black string and localized black hole branches is not shown because the available numerical code breaks in this region [34. It is however conjectured that the two branches do indeed converge into a topology-changing merger with a conical structure [35], and there is numerical evidence for this [36]. The approximations for the fluid description become invalid close to this transition point (as do, too, the gravitational ones), but certainly a topology-changing transition seems to take place when this is neglected and the calculations are pushed until pinch-off. The similarities between the two approaches are strong enough to lend credence to the existence of the transition. What seems less clear is whether the transition in the fluid involves the same conical structure as in [35, 36]. The strong gravitational interaction between the images of localized black holes close to the merger, compared to the absence of interaction between the images of fluid balls, indicates the possibility of at least differences of detail in the two cases.

An even more uncanny similarity in the phase structure of both fluid tubes and black strings refers to the critical dimension of spacetime for which the transition between uniform and nonuniform phases changes from first to second order 9 For fluid tubes ref. [20] found (and we have reproduced) that this happens at $d_{*}=11$, while for black strings it happens at $D_{*}=14$ for the microcanonical ensemble [37] and $D_{*}=13$ for the canonical ensemble [38. If we assume that, as in the AdS context, the $d$-dimensional fluids are to be related to $D=d+2$-dimensional black holes, then the apparent closeness in the values of the critical dimensions is truly startling.

The qualitative similarities between the dispersion relations in the Rayleigh-Plateau and in the Gregory-Laflamme instability have also been pointed out before for the case of static tubes and strings [1]. The parallels extend to further dynamical details, such as the fact that for static

\footnotetext{
${ }^{9}$ In this and in the next section we shall use $d$ and $D$, respectively, for the spacetime dimensions in which the fluid lumps and the black holes live.
} 
solutions, non-axisymmetric modes $(m \neq 0)$ are stable in the plasma, while the GL instability also only affects $s$-modes [29, 39]. Furthermore, a boost along the static plasma tube has the same effect as boosting the black string: it is only a kinematic effect increasing the threshold wavenumber for $s$-modes and leaving all other modes stable [29].

Rotating black strings are affected by the same instability. The GL instability in rotating black strings was analysed in [40]. They concluded that the GL instability persists for rotating black strings all the way to extremality and the threshold wavenumber increases as the rotation grows (see Fig. 1 of [40]). We could only treat small rotation analytically, but our dual plasma results, summarized in Fig. 6, agree with this: the instability persists as rotation grows and it actually gets stronger, with the critical wavenumber increasing with the rotation. In fact this can be understood intuitively. The centrifugal force scales with the radial distance, so it is larger at the crest of the non-uniformity triggered by the RP instability than at the trough. As a result the distance between the crest and trough is stretched: rotation enhances the instability. An interesting qualitatively new feature that rotation adds is that non-axisymmetric modes $(m \neq 0)$ can become unstable to the Rayleigh-Plateau instability for sufficiently large rotation.

Among the phases for rotating tubes that we have uncovered, a particularly interesting one is the pinched non-uniform tube (pNUT), since it has no analogue for static tubes. Its shape certainly suggests that we should think of it as the result of a bulge in the non-uniform tube beginning to behave like a rotating ball that develops a pinch. Since pinched black holes are also expected to exist in six-dimensional vacuum gravity (actually in any dimension larger than five) [32, 33], it is then natural to conjecture that rotating black strings in six dimensions also have phases of pinched non-uniform black strings, in addition to the already known uniform black strings, uniform black tubes (i.e., the black ring of [31] times a flat direction), and the expected more conventional non-uniform black string.

Such remarkable similitude between the dynamics of fluid tubes and of vacuum black strings makes it tempting to try to push it further in order to address an outstanding open problem: the time evolution of the GL instability and its final fate. The final stage of this evolution is not known in gravity, since the available numerical code, which has been developed only for $D=5$, breaks down for late times [41. On the other hand, the time evolution of the (non-relativistic) RP instability has been the object of several experimental and numerical studies, see e.g., [42] which considers viscous fluids in four spacetime dimensions (unfortunately, higher-dimensional fluids remain unavailable experimentally). We would expect that for static initial data, the classical and relativistic evolution should not differ substantially. It is then justified to compare the initial time evolution of the GL instability, Fig. 4 of [41], with the early stage dynamical evolution of the RP instability, e.g., Fig. 1 of [42]. There appears to be a nice match between the two. In the fluid, where the evolution has been followed until its endpoint, the uniform tube pinches-off and breaks. Starting from a single sinusoidal perturbation in a cylindrical liquid bridge (fluid tube), the higher harmonics generated by non-linear effects are responsible for the development of a long neck that breaks the tube in a self-similar process. One ends up with an array of satellite drops with different sizes [42]10.

\footnotetext{
${ }^{10}$ Actually, two main regimes have been observed depending on the viscosity of the fluid. For low viscosity, there is repeated stretching and breakup, ending with several satellite droplets. For high viscosity, a single breakup that takes a long time can occur and in the end a single satellite drop is formed.
} 
We may expect such behavior to be representative of the time evolution of the instability of fluids in subcritical dimensions $d<d_{*}=11$. A numerical study of the non-relativistic evolution for inviscid fluids in several dimensions has been made in [21. The endpoint of the RP instability does appear to differ for subcritical and supercritical dimensions. For $d<d_{*}$ the endpoint is a drop or an array of drops. Again, it is tempting to argue in favor of a similar evolution in the gravitational system, in which the black string pinches off completely and forms an array of black holes, possibly of different sizes11. The same caveats apply here as in the discussion of the ball-tube/black holeblack string transition: the fluid description breaks down near pinch-off; and blobs of fluid do not self-interact whereas gravitating horizon blobs do. However, it is already significant that pinch off does occur for viscous fluids, and that no study reveals any sign of the instability slowing down as pinch-off is approached in $d<d_{*}$.

The situation may be cleaner above the critical dimension. Ref. [21] finds that in this case the endpoint of the RP instability is a non-uniform tube with constant mean curvature. In this case there is no concern about the validity of the fluid description, since the tube can remain thick enough during its evolution. The role of viscosity is not too clear, but it might be expected to smooth the evolution. This suggests that the endpoint of the GL instability on a black string above the critical dimension could be a non-uniform black string, like ref. [4] suggested.

The non-linear GL evolution when rotation is present has not been studied yet but again, the (non-relativistic) RP evolution of fluids is well studied (see e.g., 44]): rotation introduces twisting effects in the pinch off of the tube and as rotation increases a dramatic centrifugal ejection of drops is observed (see the photographs of [44]).

Of course it is possible that black strings and black holes in vacuum gravity behave in a closely similar way to fluids only as long as one considers configurations that are stationary, slowly evolving, and/or not too non-uniform. The dynamics of vacuum gravity may depart significantly from fluid dynamics away from these regimes and furthermore, in contrast to the situation for gravity in AdS, it is not obvious whether it can be accurately pictured as fluid dynamics in some controlled approximation. The next section addresses this issue in more detail.

\section{The black hole/fluid analogy revisited}

The similarities discussed in the previous section naturally prompt the question of to what extent we can trust fluid dynamics as a guide for unknown dynamics of vacuum black holes in asymptotically flat space. In other words, since the analogy between fluids and black holes was observed in the first place for asymptotically flat black holes, is there any precise meaning to it? Clearly, we cannot just take the results for AdS black holes in the limit in which the cosmological constant vanishes. The fluid description for AdS black holes requires that their size be much larger than the AdS curvature radius, so black holes smaller than this size fall outside the scope of the dual hydrodynamics.

We have discussed a number of qualitative or semi-quantitative features that fluid lumps share with black holes in vacuum gravity. However, there is at least one crucial difference that would

\footnotetext{
${ }^{11}$ These have already been constructed in $[22]$. It is entropically favorable for these arrays of black holes to merge into a single one.
} 
appear to preclude a precise correspondence. Classical General Relativity in vacuum is a scaleinvariant theory, which implies that all properties of a black hole scale uniformly with mass. That is, all Schwarzschild black holes are essentially the same; all Kerr black holes with the same value of $J / G M^{2}$ also have the same properties; and all black strings in Kaluza-Klein space are the same when the compact radius is scaled to keep $L /(G M)^{1 /(D-2)}$ constant, e.g., the Gregory-Laflamme threshold mode wavelength for a static black string must scale like the inverse of the horizon transverse radius. This scale invariance is not present for the fluid: the surface tension sets a scale that distinguishes fluid lumps of different size: a fluid ball of a given radius is not simply a scaled-up version of a ball of half that radius - for instance, the former can more easily break up than the latter. For a large plasma ball, the relative entropy cost of breaking it in two pieces becomes arbitrarily small as the radius $R$ of the ball gets arbitrarily large. For instance, for a ball of fluid with initial entropy $S_{i}$, temperature $T_{i}$ and radius $R_{i}$, that breaks into two equal balls, each with entropy $S_{f} / 2$, temperature $T_{f}$ and radius $R_{f}$, keeping the total energy unchanged, the ratio between final and initial entropies is

$$
\left.\frac{S_{f}}{S_{i}}\right|_{\text {fluid }} \simeq \frac{T_{i}}{T_{f}}\left(1+\frac{\sigma}{\rho}\left(\frac{1}{R_{i}}-\frac{1}{R_{f}}\right)\right),
$$

where we have assumed that the ball radii are much larger than the length scale $\sigma / \rho$ set by the energy density $\rho$ and surface tension $\sigma$ of the fluid 12 . To leading order, $R_{f}^{-1}=2^{\frac{1}{d-1}} R_{i}^{-1}>R_{i}^{-1}$, and moreover, if the balls have negative specific heat the smaller final balls will be hotter than the initial one, $T_{f}>T_{i}$, with $T_{f} / T_{i}-1$ a positive and small quantity of order $\sigma /\left(\rho R_{i}\right)$. Then $S_{f}<S_{i}$, so the process is suppressed, but the relative entropy cost of breaking

$$
\left.\frac{\Delta S}{S}\right|_{\text {fluid }}=\left.\frac{S_{f}-S_{i}}{S_{i}}\right|_{\text {fluid }} \simeq-\delta_{d} \frac{\sigma}{\rho R_{i}}
$$

is small, with large fluid balls breaking more easily than smaller ones. Here $\delta_{d}$ is a positive, dimension-dependent number which, for a plasma with the equation of state considered in sec. 2.2 , is

$$
\delta_{d}=(d-1)\left(2^{\frac{1}{d-1}}-1\right) .
$$

For a Schwarzschild black hole, in contrast, the relative entropy cost for splitting into two equal black holes keeping the total mass fixed, remains constant independently of the black hole size,

$$
\left.\frac{\Delta S}{S}\right|_{\mathrm{bh}}=\left.\frac{S_{f}-S_{i}}{S_{i}}\right|_{\mathrm{bh}}=2^{-\frac{1}{D-3}}-1
$$

It is amusing that, if we make the identification $D=d+2$ that is suggested by the duality between SS-AdS $d_{d+2}$ black holes and $d$-dimensional fluid lumps, the powers of 2 in these formulas coincide. But on the other hand, the identification between (8.39) and (8.41) appears to require that $\sigma \sim \rho R$. This is not only a rather bizarre behavior for the surface tension, which grows linearly with the size of the fluid ball. It also implies that the black hole is equated with a fluid ball that is always small 13 , in the sense that surface effects are always strong so the regime (6.34) is never attained.

\footnotetext{
${ }^{12}$ To leading order we can neglect the difference in energy density in the initial and final fluid balls.

${ }^{13}$ This is indeed the case for small black holes in AdS, which resemble asymptotically flat ones.
} 
The apparent reason for this is, again, that vacuum gravity does not have a scale parameter to characterize a black hole as parametrically large.

So the crux of the problem is that we are trying to relate objects in parameter spaces of different dimensionality! For the black holes, as argued, we are always free to fix a scale so as to set the mass, or the length $(G M)^{\frac{1}{D-3}}$, equal to one, and then completely characterize the black hole by its angular momenta. For a fluid lump, the theory comes with a scale already, namely the length scale $\sigma / \rho$, so in order to specify a fluid ball we must provide its size relative to that scale (or its energy), and its angular momenta - i.e., one more parameter than for a black hole.

One may point out that a natural scale that appears in black hole physics is the Planck length, which does allow to make a distinction between small and large black holes. However, this is of no help to make a better comparison between vacuum black holes and fluid lumps, since in the limit in which the 'cutoff' scale becomes negligible, namely, when the fluid lumps or the black holes become very large relative to that scale, their properties are clearly different, as illustrated above. It would be desirable to have a way of deciding whether a black hole is large or small within classical vacuum General Relativity, but this seems hard in the absence of a classical fundamental scale.

Does this, then, imply, that there can be no limit in which the equations of the dynamics and the phase space of vacuum black holes can be mapped into the equations and phase space of a fluid, with effective parameters that satisfy

$$
\frac{\sigma}{\rho R} \ll 1 ?
$$

In the following we make some observations that suggest otherwise.

Let us first note that an obvious difference between fluids and black holes is that two disconnected lumps of fluid do not attract each other (at least within the framework in which we are considering fluids). This must obviously imply that some phenomena, like the deep non-linear evolution of the GL instability, or the mergers involving vacuum black holes or black rings, may behave differently than those for their fluid counterparts: in the former case the gravitational attraction between different lumps on the horizon presumably plays a role similar to the attraction between two black holes and thus affects their evolution 14. Thus, any limit in which vacuum black holes behave accurately as fluids must be a limit in which this gravitational attraction is suppressed.

Such a limit seems to arise if we consider gravity in a spacetime with a large number of noncompact dimensions. As the number of dimensions increases, the gravitational potential becomes steeper and more localized near the source, and flatter and weaker at larger distances. Furthermore, since the black hole entropy

$$
S \propto M^{\frac{D-2}{D-3}}
$$

is directly proportional to the mass in the limit $D \rightarrow \infty$, there is no entropy cost in splitting a black hole: (8.41) yields $\Delta S / S \rightarrow 0$ in this limit. As we observed above, this is just like for a large drop of fluid. Indeed, both for a fluid with vanishingly small surface tension and for a black hole in $D \rightarrow \infty$ the (Hagedorn-like) relation $E=T S$ is satisfied 15 .

\footnotetext{
${ }^{14}$ That these effects must, of necessity, be strongly suppressed for the class of AdS black holes that are dual to plasma lumps, is a remarkable consequence of the duality.

${ }^{15}$ For the fluid lump $E$ here is the difference between its energy and the energy of the (unconfined) vacuum it displaces.
} 
This enhanced instability of the black hole horizon to break up has a rather precise manifestation in the equivalence between the Gregory-Laflamme and Rayleigh-Plateau instabilities. Given the observations above, we can expect a black string in $D \rightarrow \infty$ to be able to split into fragments of any length, i.e., the wavelength of the threshold mode is expected to approach zero. Indeed, the GL instability in arbitrary dimension has been studied in [45], and even if the equation for the threshold mode is fairly complicated, it simplifies greatly if we expand in $1 / D$ to leading order,

$$
\chi^{\prime \prime}(r)+\frac{D}{r} \chi^{\prime}(r)-k^{2} \chi(r)=0 .
$$

This is essentially the same as the equation for the RP threshold ( $\omega=0)$ mode (5.21), and it becomes precisely the same if we identify $D \simeq n \simeq d$ for large $D$ and $d$. The wavelength of the GL threshold mode in this limit vanishes as $r_{s} / \sqrt{D}$, in accord with the expectation above.

Expanding on these observations, we can try to identify what plays the role of the dimensionless quantity $\sigma /(\rho R)$ in the gravitational side. The answer is obvious: since the only small dimensionless parameter we have at our disposal in the gravity side is $1 / D$, both must be related. A more precise mapping can be obtained by comparing (8.39) and (8.41). For large $D$, the latter becomes

$$
\left.\frac{\Delta S}{S}\right|_{\mathrm{bh}} \simeq-\frac{\log 2}{D}
$$

which leads to the identification

$$
\frac{\sigma}{\rho R} \sim \frac{1}{D}
$$

We are not necessarily proposing that the limit $d \rightarrow \infty$ must be taken in the fluid side too, but if we do, and use the value (8.40), $\delta_{d} \rightarrow \log 2$, then the identification becomes precise, $\frac{\sigma}{\rho R}=D^{-1}$, at least to leading order in $1 / D$.

Eq. (8.46) illustrates a main feature of our proposal. As we have discussed, the main problem for a fluid/black hole correspondence is the apparent absence in classical vacuum GR of a way to decide whether a black hole is intrinsically large or small. Our suggestion amounts to saying that a black hole is large or small depending on the number of spacetime dimensions it lives in. Black holes in low dimensions are to be regarded as tiny droplets, with surface energy comparable to bulk energy, whereas black holes in very high dimensions behave rather like large lumps of fluid. In other words, we are using $D$ as an additional parameter characterizing a black hole. We are making crucial use here of the observation that in classical General Relativity in vacuum the only dimensionless tunable parameter at our disposal is $D$, a point that has also been urged by B. Kol and collaborators [46].

It might be that these similarities between fluids and black holes at large $D$ only reflect purely geometrical aspects of the relation between volumes and areas at very high dimensions (note in particular that $D$, but not $\sigma /(\rho R)$, appears in (8.44) $)$. But at any rate we feel that these observations suggest that perhaps by considering a $1 / D$ expansion, and possibly accounting for the effects of gravitational interaction in this expansion, a more accurate map betwen vacuum black holes and fluids may be achieved that allows to understand not only the similarities that have been observed, but also the differences. 


\section{Acknowledgments}

We thank Vitor Cardoso, Veronika Hubeny, Umpei Miyamoto, Renaud Parentani and Toby Wiseman for interesting discussions. MMC, OJCD and RE were supported in part by DURSI 2005 SGR 00082, MEC FPA 2004-04582-C02 and FPA-2007-66665-C02, and the European Community FP6 program MRTN-CT-2004-005104. OJCD and RE thank the Niels Bohr Institute for hospitality and the organizers of the workshop "Mathematical Aspects of General Relativity", Copenhagen, April 2008; OJCD, RE and DK thank CERN for hospitality during the programme "Black Holes: A Landscape of Theoretical Physics Problems", August-October 2008; and MMC, OJCD and RE are grateful to the organizers of the workshop "Quantum Black Holes, Braneworlds and Holography", Valencia, May 2008, where part of this work was done. MMC aknowledges financial support provided by the FWO - Vlaanderen, project G.0235.05 and in part by the Federal Office for Scientic, Technical and Cultural Affairs through the Interuniversity Attraction Poles Programme Belgian Science Policy P6/11-P. OJCD acknowledges financial support provided by the European Community through the Intra-European Marie Curie contract MEIF-CT-2006-038924. This work was partially funded by FCT through project PTDC/FIS/64175/2006. DK was supported in part by INFN, MIUR-PRIN contract 20075ATT78, and by the European Community FP6 program MRTN-CT-2004-005104.

\section{References}

[1] V. Cardoso and O. J. C. Dias, "Gregory-Laflamme and Rayleigh-Plateau instabilities," Phys. Rev. Lett. 96 (2006) 181601 arXiv:hep-th/0602017.

[2] V. Cardoso, O. J. C. Dias and L. Gualtieri, "The return of the membrane paradigm? Black holes and strings in the water tap," Int. J. Mod. Phys. D 17 (2008) 505 arXiv:0705.2777 [hep-th]].

[3] V. Cardoso and L. Gualtieri, "Equilibrium configurations of fluids and their stability in higher dimensions," Class. Quant. Grav. 23 (2006) 7151 arXiv:hep-th/0610004.

[4] R. Gregory and R. Laflamme, "Black strings and p-branes are unstable," Phys. Rev. Lett. 70 (1993) 2837 arXiv:hep-th/9301052].

[5] R. Gregory and R. Laflamme, "The Instability of charged black strings and p-branes," Nucl. Phys. B 428 (1994) 399 arXiv:hep-th/9404071.

[6] S. Bhattacharyya, S. Lahiri, R. Loganayagam and S. Minwalla, "Large rotating AdS black holes from fluid mechanics," arXiv:0708.1770 [hep-th].

[7] S. Bhattacharyya, V. E. Hubeny, S. Minwalla and M. Rangamani, "Nonlinear Fluid Dynamics from Gravity," JHEP 0802 (2008) 045 [arXiv:0712.2456 [hep-th]].

[8] K. Copsey and G. T. Horowitz, "Gravity dual of gauge theory on $\mathrm{S}^{* *} 2 \times \mathrm{S}^{* *} 1 \mathrm{x}$ R," JHEP 0606, 021 (2006) arXiv:hep-th/0602003. 
[9] R. B. Mann, E. Radu and C. Stelea, "Black string solutions with negative cosmological constant," JHEP 0609 (2006) 073 arXiv:hep-th/0604205.

[10] A. Bernamonti, M. M. Caldarelli, D. Klemm, R. Olea, C. Sieg and E. Zorzan, "Black strings in $\mathrm{AdS}_{5}, "$ JHEP 0801 (2008) 061 [arXiv:0708.2402 [hep-th]].

[11] Y. Brihaye, T. Delsate and E. Radu, "On the stability of AdS black strings," Phys. Lett. B 662 (2008) 264 arXiv:0710.4034 [hep-th]].

[12] O. Aharony, S. Minwalla and T. Wiseman, "Plasma-balls in large N gauge theories and localized black holes," Class. Quant. Grav. 23, 2171 (2006) arXiv:hep-th/0507219.

[13] S. Lahiri and S. Minwalla, "Plasmarings as dual black rings," arXiv:0705.3404 [hep-th].

[14] S. Bhardwaj and J. Bhattacharya, "Thermodynamics of Plasmaballs and Plasmarings in 3+1 Dimensions," arXiv:0806.1897 [hep-th].

[15] R. Emparan and H. S. Reall, "Black Holes in Higher Dimensions," Living Rev. Rel. 11 (2008) 6 arXiv:0801.3471 [hep-th]].

[16] B. Kol, "The phase transition between caged black holes and black strings: A review," Phys. Rept. 422 (2006) 119 arXiv:hep-th/0411240.

[17] N. A. Obers, "Black Holes in Higher-Dimensional Gravity," arXiv:0802.0519 [hep-th].

[18] S. S. Gubser, "On non-uniform black branes," Class. Quant. Grav. 19 (2002) 4825, arXiv:hep-th/0110193.

[19] T. Wiseman, "Static axisymmetric vacuum solutions and non-uniform black strings," Class. Quant. Grav. 20, 1137 (2003) arXiv:hep-th/0209051.

[20] U. Miyamoto and K. i. Maeda, "Liquid bridges and black strings in higher dimensions," Phys. Lett. B 664, 103 (2008) arXiv:0803.3037 [hep-th]].

[21] U. Miyamoto, "Curvature driven diffusion, Rayleigh-Plateau, and Gregory-Laflamme," Phys. Rev. D 78 (2008) 026001 arXiv:0804.1723 [hep-th]].

[22] O. J. C. Dias, T. Harmark, R. C. Myers and N. A. Obers, "Multi-black hole configurations on the cylinder," Phys. Rev. D 76, 104025 (2007) arXiv:0706.3645 [hep-th]].

[23] K. i. Maeda and U. Miyamoto, "Black hole-black string phase transitions from hydrodynamics," arXiv:0811.2305 [hep-th].

[24] S. Bhattacharyya, R. Loganayagam, S. Minwalla, S. Nampuri, S. P. Trivedi and S. R. Wadia, "Forced Fluid Dynamics from Gravity," arXiv:0806.0006 [hep-th].

[25] M. Van Raamsdonk, "Black Hole Dynamics From Atmospheric Science," JHEP 0805 (2008) 106 [arXiv:0802.3224 [hep-th]]. 
[26] T. Young, "An essay on the cohesion of fluids," Philos. Trans. R. Soc. London 95, 65 (1805). P. S. Laplace, "Traité de Mécanique Céleste; Supplément au Dixième Livre, Sur l'Action Capillaire," (Courcier, Paris, 1806); "Supplément à la Théorie de l'Action Capillaire," (Courcier, Paris, 1806).

[27] T. Harmark and N. A. Obers, "Phases of Kaluza-Klein black holes: A brief review," arXiv:hep-th/0503020.

[28] G. T. Horowitz, "Playing with black strings," arXiv:hep-th/0205069.

[29] J. L. Hovdebo and R. C. Myers, "Black rings, boosted strings and Gregory-Laflamme," Phys. Rev. D 73 (2006) 084013 arXiv:hep-th/0601079.

[30] S. Chandrasekhar, "Hydrodynamic and Hydromagnetic Stability," Dover, New York, 1981.

[31] R. Emparan and H. S. Reall, "A rotating black ring in five dimensions," Phys. Rev. Lett. 88 (2002) 101101 arXiv:hep-th/0110260].

R. Emparan and H. S. Reall, "Black rings," Class. Quant. Grav. 23 (2006) R169 arXiv:hep-th/0608012].

[32] R. Emparan and R. C. Myers, "Instability of ultra-spinning black holes," JHEP 0309 (2003) 025 arXiv:hep-th/0308056.

[33] R. Emparan, T. Harmark, V. Niarchos, N. A. Obers and M. J. Rodriguez, "The Phase Structure of Higher-Dimensional Black Rings and Black Holes," JHEP 0710 (2007) 110 arXiv:0708.2181 [hep-th]].

[34] H. Kudoh and T. Wiseman, "Connecting black holes and black strings," Phys. Rev. Lett. 94 (2005) 161102, arXiv:hep-th/0409111.

[35] B. Kol, "Topology change in general relativity and the black-hole black-string transition," JHEP 0510, 049 (2005) arXiv:hep-th/0206220.

[36] B. Kol and T. Wiseman, "Evidence that highly non-uniform black strings have a conical waist," Class. Quant. Grav. 20, 3493 (2003) arXiv:hep-th/0304070.

[37] E. Sorkin, "A critical dimension in the black-string phase transition," Phys. Rev. Lett. 93 (2004) 031601, arXiv:hep-th/0402216.

[38] H. Kudoh and U. Miyamoto, "On non-uniform smeared black branes," Class. Quant. Grav. 22 (2005) 3853, arXiv:hep-th/0506019.

[39] H. Kudoh, "Origin of black string instability," Phys. Rev. D 73 (2006) 104034 arXiv:hep-th/0602001.

[40] B. Kleihaus, J. Kunz and E. Radu, "Rotating nonuniform black string solutions," JHEP 0705 (2007) 058 arXiv:hep-th/0702053. 
[41] M. W. Choptuik, L. Lehner, I. Olabarrieta, R. Petryk, F. Pretorius and H. Villegas, "Towards the final fate of an unstable black string," Phys. Rev. D 68 (2003) 044001 arXiv:gr-qc/0304085.

[42] M. Tjahjadi, H. A. Stone and J. M. Ottino, "Satellite and subsatellite formation in cappilary breakup," J. Fluid Mech. 243 (1992) 297.

[43] G. T. Horowitz and K. Maeda, "Fate of the black string instability," Phys. Rev. Lett. 87 (2001) 131301 arXiv:hep-th/0105111.

[44] J. P. Kubitschek and P. D. Weidman, "Helical instability of a rotating viscous liquid jet," Phys. Fluids 19 (2007) 114108.

[45] B. Kol and E. Sorkin, "On black-brane instability in an arbitrary dimension," Class. Quant. Grav. 21 (2004) 4793 arXiv:gr-qc/0407058.

T. Harmark, V. Niarchos and N. A. Obers, "Instabilities of black strings and branes," Class. Quant. Grav. 24 (2007) R1 arXiv:hep-th/0701022.

[46] V. Asnin, D. Gorbonos, S. Hadar, B. Kol, M. Levi and U. Miyamoto, "High and Low Dimensions in The Black Hole Negative Mode," Class. Quant. Grav. 24 (2007) 5527 arXiv:0706.1555 [hep-th]]. 